\title{
Innovative financing through pay-for-performance for providers to improve quality of care in Bangladesh: Transforming research into action
}

Md. Noorunnabi Talukder

Population Council

Ubaidur Rob

Population Council

Laila Rahman

Population Council

Ismat Ara Hena

Population Council

Follow this and additional works at: https://knowledgecommons.popcouncil.org/departments_sbsr-rh

Part of the Demography, Population, and Ecology Commons, Family, Life Course, and Society Commons, Health Policy Commons, International Public Health Commons, Maternal and Child Health Commons, Public Health Education and Promotion Commons, and the Women's Health Commons How does access to this work benefit you? Let us know!

\section{Recommended Citation}

Talukder, Md. Noorunnabi, Ubaidur Rob, Laila Rahman, and Ismat Ara Hena. 2011. "Innovative financing through pay-for-performance for providers to improve quality of care in Bangladesh: Transforming research into action," workshop report. Dhaka: Population Council. 


\section{INNOVATIVE FINANCING THROUGH PAY-FOR-PERFORMANCE FOR PROVIDERS TO IMPROVE QUALITY OF CARE IN BANGLADESH}

TRANSFORMING RESEARCH INTO ACTION

MD. NOORUNNABI TALUKDER UBAIDUR ROB

LAILA RAHMAN ISMAT ARA HENA 


\section{INNOVATIVE FINANCING THROUGH PAY-FOR-PERFORMANCE FOR PROVIDERS TO IMPROVE QUALITY OF CARE IN BANGLADESH}

\section{TRANSFORMING RESEARCH INTO ACTION}

Md. Noorunnabi Talukder

Ubaidur Rob

Laila Rahman

Ismat Ara Hena

Population Council, Bangladesh

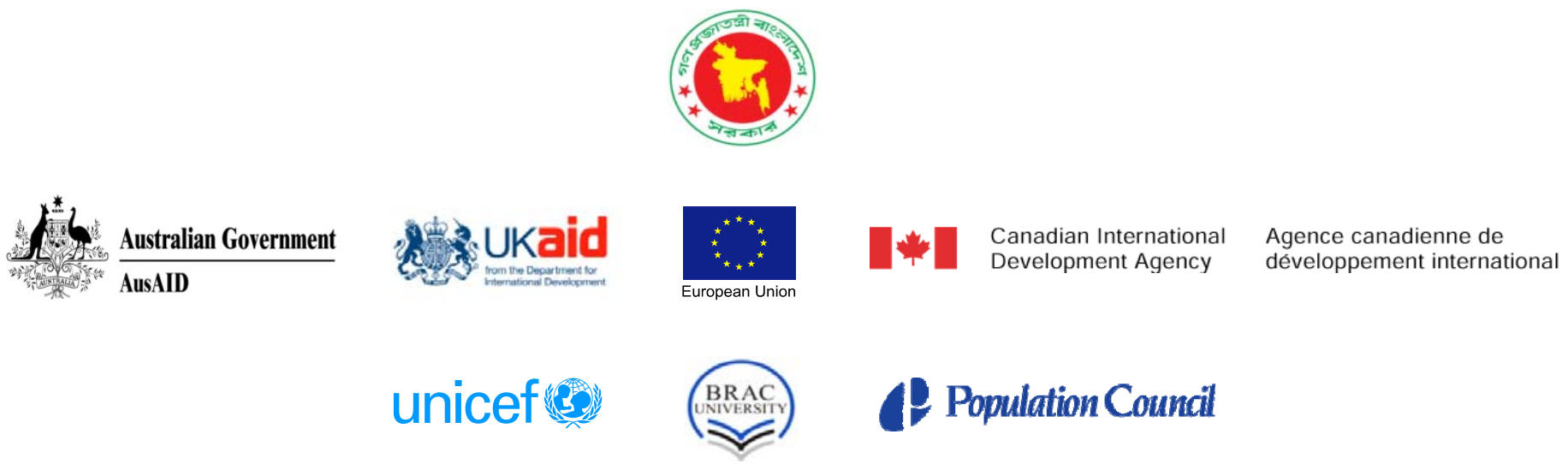




\section{(1) Population Council}

The Population Council confronts critical health and development issues-from stopping the spread of HIV to improving reproductive health and ensuring that young people lead full and productive lives. Through biomedical, social science, and public health research in 50 countries, we work with our partners to deliver solutions that lead to more effective policies, programs, and technologies that improve lives around the world. Established in 1952 and headquartered in New York, the Council is a nongovernmental, nonprofit organization governed by an international board of trustees.

Population Council

Bangladesh Country Office

House CES (B) 21, Road 118, Gulshan, Dhaka, Bangladesh

\section{www.popcouncil.org}

Suggested citation: Md. Noorunnabi Talukder, Ubaidur Rob, Laila Rahman, and Ismat Ara Hena. 2011. "Innovative financing through pay-for-performance for providers to improve quality of care in Bangladesh: Transforming research into action," Workshop Report. Dhaka: Population Council.

(C) 2011 The Population Council, Inc. 


\section{TABLE OF CONTENTS}

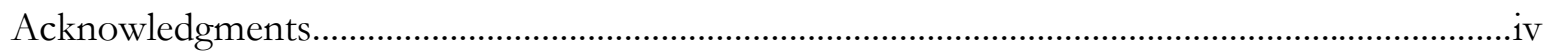

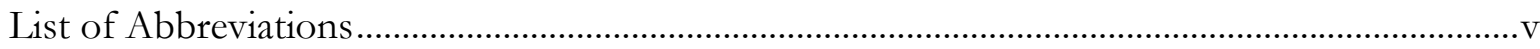

Executive Summary ………….......................................................................................................

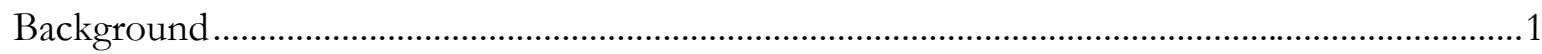

Purpose and Organization of the Workshop.............................................................................

Performance-Based Financing: Theory and International Experiences .......................................5

Performance-Based Financing in Bangladesh: Policies and Experiences.....................................

Defining Future Performance-Based Financing Model: Findings from Group Work...............19

Prospects and Challenges of Performance-Based Financing: Stakeholders' Views ....................25

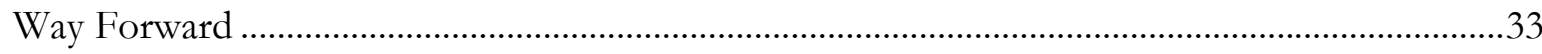

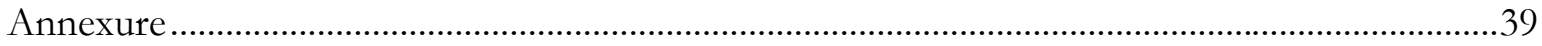




\section{ACKNOWLEDGMENTS}

This report is the outcome of a two-day workshop on "Innovative financing through pay-forperformance for providers to improve quality of care in Bangladesh: Transforming research into action" organized by Directorate General of Health Services (DGHS), in partnership with the Health Economics Unit, Ministry of Health and Family Welfare (MOHFW). Population Council in collaboration with United Nations Children's Fund (UNICEF) provided technical assistance to organize the workshop. The Council expresses sincere gratitude to Australian Agency for International Aid, UK Department for International Development, European Union and Canadian International Development Agency for their financial contribution through UNICEF in carrying out the workshop.

Population Council is grateful to Mr. Md. Humayun Kabir, Secretary, MOHFW, who was present at the workshop as the Chief Guest. We are indebted to Dr. Md. Shamsul Haque, Director, Primary Health Care and Line Director, Maternal, Newborn, Child and Adolescent Health, DGHS for chairing the workshop. We would like to extend our heartfelt thanks to Mr. Prasanta Bhushan Barua, Joint Chief, Health Economics Unit, MOHFW, Dr. Mohammed Sharif, Director, Maternal and Child Health Services and Line Director, Maternal Child-Reproductive Health, Directorate General of Family Planning, and Mr. Michel Saint-Lot, Deputy Representative, UNICEF for their presence and contribution as Special Guests at the workshop. The Council also expresses sincere thanks to Dr. A.B.M. Jahangir Alam, former Director, Primary Health Care, DGHS and Dr. Saleh Mohammad Rafique, former Director, Primary Health Care, DGHS for moderating group sessions.

We particularly acknowledge the contribution made by the presenters of the workshop: Dr. Ubaidur Rob, Country Director, Population Council; Mr. Prasanta Bhushan Barua, Joint Chief, Health Economics Unit, MOHFW; Dr. Azizul Alim, Deputy Program Manager, DGHS, MOHFW; Dr. Riad Mahmud, Health Officer, UNICEF/Bangladesh; Ms. Laila Rahman, Senior Program Officer, Population Council; Dr. S.M. Shahida, Assistant Professor (Gynecology/Obstetrics), Mymensingh Medical College; and Dr. Md. Feroze Khan, Upazila Health and Family Planning Officer, Islampur Upazila Health Complex, Jamalpur. We gratefully acknowledge the strong participation of managers and consultants of 12 health facilities from Jamalpur, Gaibandha and Kurigram districts under Payfor-Performance project and 11 Upazila Health Complexes under Demand-side Financing scheme. We sincerely thank the participants who attended the workshop, including faculty from Mymensingh Medical College and Rangpur Medical College.

Special thanks go to Population Council staff members for their sincere efforts in organizing this event. Technical assistance from Dr. Md. Sayedur Rahman, Program Manager, DGHS and Dr. Riad Mahmud, Health Officer, UNICEF/Bangladesh is greatly appreciated. We are indebted to Mr. A.K.M. Zafar Ullah Khan, Advisor, Population Council for his continued support at every step of organizing the workshop and for reviewing the draft version of this report. 


\section{LIST OF ABBREVIATIONS}

\begin{tabular}{|c|c|}
\hline ANC & Antenatal care \\
\hline $\mathrm{BCC}$ & Behavior change communication \\
\hline BMA & Bangladesh Medical Association \\
\hline CSBA & Community skilled birth attendant \\
\hline DGFP & Directorate General of Family Planning \\
\hline DGHS & Directorate General of Health Services \\
\hline DSF & Demand-side financing \\
\hline EmOC & Emergency obstetric care \\
\hline GOB & Government of Bangladesh \\
\hline $\mathrm{HEF}$ & Health Equity Fund \\
\hline HFWC & Health and Family Welfare Center \\
\hline $\mathrm{HIV} / \mathrm{AIDS}$ & Human immunodeficiency virus/Acquired immune deficiency syndrome \\
\hline HPNSDP & Health Population and Nutrition Sector Development Program \\
\hline MCWC & Mother and Child Welfare Center \\
\hline MDG & Millennium Development Goal \\
\hline MNCAH & Maternal, newborn, child and adolescent health \\
\hline $\mathrm{MNCH}$ & Maternal, newborn and child health \\
\hline $\mathrm{MNH}$ & Maternal and newborn health \\
\hline MOHFW & Ministry of Health and Family Welfare \\
\hline NGO & Non-governmental organization \\
\hline NHI & National Health Insurance \\
\hline OGSB & Obstetrical and Gynecological Society of Bangladesh \\
\hline P4P & Pay-for-performance \\
\hline PBF & Performance-based financing \\
\hline $\mathrm{PNC}$ & Postnatal care \\
\hline QAG & Quality assurance group \\
\hline
\end{tabular}




$\begin{array}{ll}\text { QAT } & \text { Quality assurance team } \\ \text { QOC } & \text { Quality of care } \\ \text { RMO } & \text { Resident Medical Officer } \\ \text { SBA } & \text { Skilled birth attendant } \\ \text { UHC } & \text { Upazila Health Complex } \\ \text { UHFPO } & \text { Upazila Health and Family Planning Officer } \\ \text { UN } & \text { United Nations } \\ \text { UNFPA } & \text { United Nations Population Fund } \\ \text { UNICEF } & \text { United Nations Children's Fund } \\ \text { USAID } & \text { U.S. Agency for International Development } \\ \text { WHO } & \text { World Health Organization }\end{array}$




\section{EXECUTIVE SUMMARY}

Access to facility-based obstetric and newborn care and improving skilled birth attendance at delivery are vital to improve maternal and neonatal health. Traditionally, underutilization of existing capacity, specifically the low use of facilities for maternal and newborn care services in rural areas, has been a critical challenge to the health system in Bangladesh. At the demand side, cost is one of the main determinants of low utilization of professional maternity care. On the other hand, due to shortage and sub-optimal performance of service providers, health facilities at the upazila level and below operate at less than their capacity. To improve access to and use of facility-based obstetric and newborn care, the Government of Bangladesh is implementing two innovative performancebased financing programs, namely demand-side financing (DSF) and pay-for-performance (P4P).

Both DSF and P4P schemes are designed to contribute to achieving the Millennium Development Goal (MDG) targets 4 and 5. Both programs contributed to the increase in institutional deliveries, yet, the country is facing challenges to expedite progress towards achieving MDG targets and to improve quality in health care services. DSF and P4P are implemented in parallel with their own merits and limitations, yet there is scope for modifications. With the purpose to identify the lessons learned, limitations of P4P and DSF models, and scopes for cross learning, a two-day workshop was organized in Dhaka. The workshop was also intended to develop recommendations for the Ministry of Health and Family Welfare (MOHFW) to incorporate changes into the DSF scheme or to modify the P4P approach for further expansion.

\section{DSF scheme at a glance}

DSF scheme provides financial support to poor women for receiving safe delivery services in rural areas. Started in 2006, currently the program is implemented in 53 upazilas. This program focuses primarily on Upazila Health Complexes, with referral to Maternal and Child Welfare Center or District Hospital. In selected upazilas, some union-level health facilities are included in the DSF program. Both demand and supply sides are financially benefited through the DSF program. At the demand side, if deliveries are conducted at the facility or by skilled birth attendant (SBA), poor pregnant women receive financial assistance to receive service. At the supply side, service providers receive case-based incentive. Incentives are given only to providers, not to management, administrative and support staff. Provider performance has been linked with quantity of services alone. Incentives are provided monthly, calculating the total number of services provided to poor pregnant women.

\section{P4P model at a glance}

P4P project is a human resource innovation initiative - paying an incentive to the institution for achieving pre-determined targets. Three District Hospitals and nine Upazila Health Complexes tested P4P approach for 14 months. At the supply side, incentives are provided to the institution, where managers, direct and indirect providers related to maternal, newborn and child health 
$(\mathrm{MNCH})$ services, and administrative and support staff are entitled to receive incentive if the performance targets are achieved or exceeded. Within the facility, quality assurance teams (QATs) have been formed for different service units to monitor and review respective performance routinely and to coordinate the activities between team members. Quarterly targets for MNCH services are set for the institution as a whole, which takes into account both quantity and quality of services. Incentives payable to providers and staff are calculated on the basis of their level of efforts. Quality assurance group (QAG), consisting of specialists from nearby higher-level hospital (Medical College Hospital or District Hospital) and professional body, in consultation with respective facility-based project implementing committee, determines performance targets, performance achievements, and eligibility for incentive. P4P uses a systematic financial reimbursement mechanism for calculating and verifying incentives. Incentive payment related to performance is reimbursed by the third party. Meanwhile, an audit firm validates the disbursement of fund.

\section{Modification of DSF and P4P is necessary}

Although financial incentive in the public sector is a new phenomenon in Bangladesh, the necessity for introducing performance-based incentive has been justified through the positive results of both DSF and P4P programs. Both DSF and P4P have the same goal but they differ in approaches and outcomes. The key difference of these two initiatives is that P4P implemented a "quality of care"based incentive mechanism for MNCH care. The workshop resulted in several recommendations to modify DSF and P4P schemes.

\section{A. Suggested modifications for DSF scheme}

\section{Incentive beneficiary}

At the supply side, along with providers working in the obstetric unit, service providers working at other units should be included as beneficiary. Whole institution approach should be adopted, i.e., all units of a facility should be brought under the umbrella of DSF. Incentives should be given to motivate service providers at the institution level, instead at the individual level. In other words, incentive should be provided to both direct and indirect providers as well as managers and administrative and support staff. At the demand side, DSF should target neonate and under-five children's services in addition to existing services for pregnant women.

\section{Performance measurement system}

In DSF, only quantity (number of services provided to the voucher clients) is considered to measure the performance of providers. Provider performance should be linked with both quantity and quality of services. Inclusion of quality as a measurement indicator in DSF scheme is urgently required. DSF needs to introduce a systematic quality assurance mechanism to measure the performance of facilities on a regular basis. 


\section{Quality assurance system}

Quality assurance system implemented under P4P project is useful in identifying gaps in service delivery, and providing mentoring on a regular basis. DSF scheme can adopt the P4P experiences of introducing both external and internal quality assurance system. For external quality assurance system, DSF needs to form "quality assurance group" within each district, where faculty from Medical College or consultants from District Hospital will visit Upazila Health Complex with comprehensive emergency obstetric care and consultants from District Hospital for Upazila Health Complex with basic emergency obstetric care. As part of internal quality assurance system, unitbased "quality assurance teams" can be formed to ensure coordination between team members and to supervise the quality within the facility.

\section{Incentive payment mechanism}

Performance should be measured quarterly replacing the current monthly measurement system. There are several problems with payment mechanism which need immediate attention:

- Increase amount of benefits for both providers and service recipients.

- Introduce referral fees for SBA to promote institutional delivery.

- Provide financial benefits to SBA for performing delivery at home.

\section{B. Suggested modifications for P4P model}

\section{Incentive beneficiary}

To sustain performance momentum, it is critical to establish reward system for outstanding performer along with the existing incentive mechanism. Field-level supervisors, e.g., assistant health inspector, health inspector, and family planning inspector, should be incorporated as beneficiary in addition to the existing fieldworkers. At the demand side, providing financial assistance to poor clients is strongly recommended. There should be a single, combined strategy in P4P approach both demand and supply side incentives should be provided. A whole district approach, incorporating all Upazila Health Complexes of a district, is required for the referral system to work effectively.

\section{Performance measurement system}

At present, the family planning unit of a facility automatically becomes eligible to receive the incentive once the health unit achieves the performance targets. It is urgently required to introduce separate targets for the family planning unit.

Services for which quantitative targets are set for the facility to receive incentive include: antenatal check-up, normal delivery, postnatal check-up, cesarean section delivery, and management of complications for newborn and under-five children. Additionally, inclusion of new indicators, e.g., number of deaths, eclampsia management, removal of retained placenta, management of ante- and post-partum hemorrhage, dilation and curettage, and post-abortion care is strongly recommended. 
Empowering facility managers is critical so that they can reward the outstanding performer and adopt corrective measure for non-performers. The size of the project implementing committee (led by facility head) should be increased by including Consultants in Obstetrics, Pediatrics, and Anesthesiology of the respective facility as its members.

\section{Quality assurance system}

The uniqueness of $\mathrm{P} 4 \mathrm{P}$ project lies in its quality assurance system. However, expanding the quality assurance system at all upazilas will be a big challenge due to limited availability of experts from tertiary-level hospitals. In the interim period, a QAG may be adopted for certain number of facilities. Alternatively, there can be quality supervision from Medical College to District Hospital and from District Hospital to Upazila Health Complex. Existing rate of honorarium for the QAG members for visiting health facilities should be increased by considering the distance and the opportunity cost.

P4P pioneered in implementing internal quality supervision by forming "quality assurance teams" for different service units within the facility. It is a positive development since QAT meetings ensure coordination and cooperation among the team members. QAT meetings should be held more frequently, e.g., weekly meeting for QATs, as a way to sustain teamwork and ensure accountability.

\section{Programmatic challenges}

Although both DSF and P4P have shown promise in rapid reduction of maternal deaths, they face challenges of sustainability. These programs are still in trial phase and highly valued for their contribution towards increasing institutional delivery. Nevertheless, human resources, sustainable funding and delegation of authority remain as the key challenges.

\section{Human resources}

Incentive may be counterproductive if required human resources, training and monitoring are not in place. In such cases, money will not lead to willingness. Highest importance should be given to ensure obstetrician and anesthesiologist pair at Upazila Health Complex; otherwise it will be difficult for DSF and P4P programs to generate significant impact. For ensuring the availability of anesthesiologist alongside obstetrician, it is important to continue emergency obstetric care training, train more anesthesiologists, and ensure no transfer or deputation during project period, without having appropriate substitute.

\section{Alternative health care financing}

Sustainability of performance-based financing programs, which are supported by development partners, is a critical challenge. Performance-based financing is not an alternative approach to health financing, as it does not generate any revenue, rather it requires a large investment. The government is not capable of supporting such projects from its own resources, for which "user fees" can be introduced as an alternative source of financing. In such cost-sharing mechanism, poor families in 
rural areas should not only be exempted from user fees, but also be given financial assistance in seeking health care.

Resource mobilization through community involvement can also be an effective tool for sustaining a program. Reactivating health management committees at different tiers of health-system service delivery may prompt a sense of ownership among community people, if health programs are jointly managed by the government and local people. If DSF and P4P could reactivate health management committees, the community might own those programs in future.

\section{Delegation of authority}

Central management requires a long process to undertake recruitment and procurement for the health facilities in rural areas. As an interim measure, district health manager (Civil Surgeon) should be allowed to rearrange the workforce across Upazila Health Complexes, so that every Upazila Health Complex will have minimum required staff-mix. Concurrently, sub-district health manager (Upazila Health and Family Planning Officer) should be empowered to undertake local recruitment and discipline staff for improving performance of the facility. Delegating and empowering local managers to keep and spend the user fees that they collect rather than return them to the state treasury is also necessary for sustainable improvement of quality of services.

\section{What next}

Performance-based incentive mechanism is an innovative strategy to tackle issues related to service use and provider performance. Evidently, both DSF and P4P are positive developments. However, these are a transitional mechanism to expedite health results. In Bangladesh, the need for continuing performance-based financing programs to meet MDGs and other health indicators is beyond argument. Specifically, such encouragement is required until certain level of institutional deliveries and improvement in maternal and child health are attained. It is urgently required to decide in what capacity performance-based financing program should continue.

Whatever the approach may be, the necessity of "quality of care" approach to improve maternal, newborn and child health services tested under the P4P scheme cannot be denied for the improvement of the monitoring of health service delivery in Bangladesh. DSF scheme can better perform by adopting such systematic quality assurance mechanism. It will be more useful and feasible if policymakers and program managers adopt results from both DSF and P4P and advance with a revised model, which will require less money and be more effective. The government needs to prepare itself to continue performance-based incentive programs in pursuance of achieving MDGs of reducing maternal and neonatal mortality. 


\section{BACKGROUND}

Access to facility-based basic and emergency obstetric and newborn care and improving skilled birth attendance at delivery are vital to improve maternal and neonatal health. Traditionally, underutilization of existing capacity, specifically the low use of facilities for maternal and newborn care services in rural areas, has been a critical challenge to the health system in Bangladesh. At the demand side, cost is one of the main determinants of low utilization of professional maternity care in rural areas. On the other hand, due to shortage and sub-optimal performance of service providers, and dysfunctional incentive environment in the health system, health facilities at the upazila level and below operate at less than their capacity. Considering the demand and supply side barriers to maternal and child health services, the Directorate General of Health Services (DGHS), Ministry of Health and Family Welfare (MOHFW), Government of Bangladesh (GOB) has undertaken a demand-side financing (DSF) program in 2006 which provides financial incentives to poor clients and maternal health providers. The DSF has suggested increase in institutional deliveries by around two and half times (Schmidt et. al. 2010 ${ }^{1}$ ). However, increased demand for services has been reported without addressing the quality of care, and appropriate expansion and facility improvement (GTZ 2008²). Specifically, the quality of health care services was not addressed by the DSF mechanism due to an environment lacking the pre-requirements for competition. Quality assurance mechanisms need to be put in place in the DSF scheme (Schmidt et. al. 2010 ${ }^{1}$ ).

On this backdrop, in 2010, DGHS has begun to implement a pilot study entitled, "Introducing a Pay-For-Performance (P4P) Approach to Increase Utilization of Maternal, Newborn and Child Health Services in Bangladesh." Performance-based incentives are awarded to a team of maternal, newborn and child health (MNCH) managers, providers and administrative and support staff of 12 public-sector health facilities. Population Council provided technical assistance to the DGHS in implementing study. The School of Public Health of BRAC University collaborated with Population Council in the study. The P4P pilot project is nested within the GOB-United Nations (UN) MNCH and maternal and newborn health $(\mathrm{MNH})$ projects. The intervention activities of the P4P project ended in November 2011. Due to the P4P interventions, service volume increased remarkably, where each District Hospital doubled the performance in institutional delivery while each Upazila Health Complex tripled that performance.

Both P4P and DSF schemes are designed to contribute to achieving the Millennium Development Goal (MDG) targets 4 and 5. However, P4P is different from DSF in three important aspects. P4P targets neonates and under-five children's services in addition to the services for pregnant women, while DSF targets only poor pregnant women. Provider incentives are calculated based on both

\footnotetext{
${ }^{1}$ Schmidt, J.O., T. Ensor, A. Hossain, and S. Khan. 2010. "Voucher as demand side financing instruments for health care: A review of the Bangladesh maternal voucher scheme.” Health Policy, 96(2): 98-107.

${ }^{2}$ GTZ. 2008. "Workshop on problems and prospects of demand side financing with reference to the maternal voucher scheme in Bangladesh." Workshop Report. Dhaka: GTZ.
} 
quantity and quality of care in the P4P project unlike the DSF scheme, which offers incentives based on the number of services provided to the voucher clients. The key difference between these two initiatives is that P4P implemented a quality of care (QOC)-based incentive mechanism for MNCH care, which could have lessons for result-based financing and other health sector financial reform initiatives.

In order to achieve MDGs 4 and 5, the GOB is poised to scale up the DSF scheme to 153 upazilas by 2015, and implement result-based financing in Bangladesh. The Health Population and Nutrition Sector Development Program (HPNSDP) 2011-2016 also outlines the importance of incorporating a "quality of care" framework; and is committed to develop a "national health care financing strategy" that would guide the nation in adopting more effective channeling of public resources towards achievement of better outcomes for the poor, women, children, disabled persons and older people.

Both DSF and P4P programs contributed to the increase in institutional deliveries, yet, the country is facing challenges to improve quality in health care services. The lessons learned from the P4P pilot may assist the government to meet its commitment of offering financing options and employing a QOC framework for MNCH care and to incorporate changes into the DSF scheme to address quality of care in providing maternal health services. DSF and P4P are implemented in parallel with their own merits and limitations, yet there is scope for modifications. Against this context, DGHS, in partnership with the Health Economics Unit of MOHFW and in technical assistance from Population Council and United Nations Children's Fund (UNICEF), organized a two-day workshop. 


\section{PURPOSE AND ORGANIZATION OF THE WORKSHOP}

The goal of the workshop was to enrich the awareness on national, regional and global experiences on performance-based incentives for providers and to shape the future performance-based financing programs to improve maternal, newborn and child health in Bangladesh. The specific objectives of the workshop were to:

- Provide an understanding of the key concepts and issues regarding the performance-based financing frameworks in the global and regional context.

- Discuss the performance-based financing initiatives including DSF and P4P schemes implemented by the Government of Bangladesh.

- Identify the lessons learned, limitations of P4P and DSF models, and scope for cross learning, and discuss issues for future implementation of performance-based financing schemes.

- Draft recommendations for the DSF scheme to incorporate the "quality of care" framework and modify its financing mechanism; recommendations for the modification of P4P model.

\section{Organization}

The workshop on "Innovative financing through pay-for-performance for providers to improve quality of care in Bangladesh: Transforming research into action" was held on 12-13 December 2011 in Dhaka. This workshop was organized by DGHS, in partnership with the Health Economics Unit, MOHFW with technical assistance from Population Council and UNICEF, and with financial assistance from Australian Agency for International Aid, UK Department for International Development, European Union and Canadian International Development Agency through UNICEF.

In the workshop, Mr. Md. Humayun Kabir, Secretary, MOHFW, was present as the Chief Guest. Dr. Md. Shamsul Haque, Director, Primary Health Care and Line Director, Maternal, Newborn, Child and Adolescent Health (MNCAH), DGHS chaired the workshop. Mr. Prasanta Bhushan Barua, Joint Chief, Health Economics Unit, MOHFW, Dr. Mohammed Sharif, Director, Maternal and Child Health Services and Line Director, Maternal Child-Reproductive Health, Directorate General of Family Planning, and Mr. Michel Saint-Lot, Deputy Representative, UNICEF were special guests at this workshop.

First day of the workshop focused on the understanding of performance-based financing approaches; and learning the results and lessons from the P4P pilot project. The workshop started with the welcome speech by Dr. Md. Shamsul Haque, Director, Primary Health Care and Line Director, MNCAH, DGHS. In the technical session, national and international experiences on performance-based financing were shared, where four papers were presented which set stage for 
discussion on the P4P pilot project in the broader context of health system financing in Bangladesh. The first presentation discussed the theory and examples on international performance-based financing experiences. The second one discussed the process of developing "Health Care Financing Strategy" in Bangladesh. The third presentation was on the DSF scheme, and the fourth centered on the P4P initiative. The session following the presentations was dedicated to testimonies presented by implementers of the P4P project.

The second day of the workshop focused on translating the learning from DSF and P4P schemes to develop recommendations. The day was primarily dedicated to group work. Two groups were formed and given specific performance-based financing programs (DSF and P4P) to compile their understanding of those initiatives. In the concluding session, two groups presented their recommendations on the issues assigned to them. After an interactive discussion, chief guest, chair and distinguished speakers of the workshop provided important directions to modify DSF or P4P for MNCH services in Bangladesh.

A total of 124 participants attended the first day of the workshop while in the second day 95 participants were present. Participants were primarily from the Directorate General of Health Services, the Directorate General of Family Planning and the Health Economics Unit of MOHFW. Managers and consultants of 12 health facilities from P4P districts and 11 DSF Upazila Health Complexes participated at the workshop. Selected faculty from Mymensingh Medical College and Rangpur Medical College were also present at the workshop. In addition, representatives from development partners and academic and professional institutions attended the workshop. 


\section{PERFORMANCE-BASED FINANCING: THEORY AND INTERNATIONAL EXPERIENCES}

This section summarizes the presentation made by Dr. Ubaidur Rob, Country Director, Population Council, through which a conceptual basis of performance-based financing (PBF) was provided and PBF experiences of several countries were shared.

\section{Why performance-based financing}

Prior to discussing performance-based financing, Dr. Rob mentioned the grounds for "paying for results" to gain attention in global health. First, there is widespread global concern that 2015 health MDGs will not be met since health investments are not producing desired health outcomes, such as reduced maternal mortality and neonatal mortality. In many developing countries, there is longstanding remuneration problem with concerns about quality and performance where providers are paid according to civil service rates. Dr. Rob referred to performance-based financing as one of the solutions to address the dysfunctional incentive environment in the health systems. At the institution level, performance-based financing is needed because it links financial investment to health results. At the individual level, providers are rewarded for improving the quality of work and increasing the quantity of services.

\section{Performance-based financing and its approaches}

Dr. Rob elaborated on the merits of PBF as a powerful health systems strengthening strategy in global health. PBF strategically uses a portion of the new resources available for health where payment is made not just for inputs but also for health outputs and outcomes. In other words, incentive (generally financial) is given to reward the attainment of positive health results. Dr. Rob quoted a definition of P4P as... "the transfer of money or material goods conditional on taking a measurable action or achieving a predetermined performance target" (Eichler $2006^{3}$ ).

PBF is a new phenomenon in the health sector of developing countries. Dr. Rob provided a concise description of PBF approach by focusing on its key characteristics.

- PBF uses money and other material goods to influence the actions of those who are delivering and receiving health services.

- Payment is dependent on performance assessed against one or more defined measures.

- Incentives may be at both individual and collective levels.

\footnotetext{
3 Eichler R. 2006. "Can Pay-for-Performance increase utilization by the poor and improve the quality of health services?" Discussion paper for the first meeting of the Working Group on Performance-Based Incentives. Washington, DC: Center for Global Development.
} 
- Incentive schemes can be developed for both supply and demand sides:

- On the supply side, incentives tie health facility bonuses to the achievement of key performance targets.

- On the demand side, households are given cash incentives to receive specific health care services.

\section{International experiences on performance-based financing}

Many developing countries are piloting or scaling up PBF programs to meet MDGs and other health indicators. Impressive gains have been observed in some applications of PBF across countries. Each country's experience with PBF is different, but by sharing approaches and lessons learned, stakeholders will better understand the processes and challenges involved in PBF program design, implementation, and scale-up. Dr. Rob referred to several global experiences with performancebased financial incentives that contributed to improved performance of providers. In particular, he provided information on the operation of performance-based financing in Rwanda, Belize, Haiti, Cambodia, and Afghanistan.

Rwanda is one of the pioneers of performance-based financing. In Rwanda, poor performance of the health system as measured by the decline in use of key health services has prompted the government to introduce PBF approach in both public and private nonprofit health facilities. Rwanda institutionalized performance-based financing in 2005 by adopting it as a national policy. In this system, incentives reward both quantity and quality of curative, maternal and child health, and HIV/AIDS services. Payment is determined by fees for selected priority services multiplied by the volume delivered and adjusted by a quality score. Therefore, health facilities can raise revenues by increasing the quantity of specified services and by improving quality.

With the goal to increase access, improve the quality of services, and enhance the productivity of health care workers, Belize is testing a supply-side P4P scheme for public and private primary health care clinics, which consists of monthly capitation payments plus annual performance award (equivalent to 10 percent of total annual earning) to the contracted clinic. Health clinics must fulfill monthly and annual performance indicators to earn their incentive payments. The scheme focuses on pre- and postnatal care and deliveries, and primary care for chronic illnesses such as diabetes, hypertension, and asthma. Belize's National Health Insurance (NHI) administers the P4P scheme and the Ministry of Health determines policies that include defining the packages of services and licensing and accrediting health facilities. NHI makes the incentive payments to each clinic, while clinic administration distributes the payment, with the independence to spend payment for incentive for providers and/or for investing in infrastructure or other inputs. The program started as a pilot in 2001 and currently covers 40 percent of the population.

Pay-for-performance in Haiti is part of a package of interventions in a bilateral health project funded by the U.S. Agency for International Development (USAID). Starting in 1999, payment to contracted nongovernmental organizations (NGOs) changed from simple reimbursement for 
documented expenditures to payment conditional on targets being reached. This performance-based payment system pays a fixed-price subcontract plus a bonus linked to attainment of specific performance targets. Remarkable improvements in key health indicators have been achieved over the years that payment for performance has been phased in. Performance-based NGOs provide essential health services to about 2.7 million people. On average, performance-based NGOs in each contract period performed better than expenditure-based NGOs. Possible future enhancements of the project include introducing performance-based payments for the public sector and experimenting with incentives tied to both HIV and tuberculosis care.

Cambodia introduced performance incentive contracts in three provinces in 2004 to address two main human resource problems in the health sector: (i) low salaries and (ii) staff reward and sanction system is not functional. Along with staff incentives, the contract introduced a Health Equity Fund (HEF) and supported training, behavior change communication (BCC) and quality improvement. Contracts were given to institutions not to individual staff, while institution contracted with individual staff. In the contracted facilities, flexible attendance (half time or full time) was allowed. Combined with other strategies such as HEF, training, BCC and quality improvement, the Performance Based Incentive Scheme contributed significantly to the motivation of the health personnel and hence to increased utilization of health services and coverage rates.

In Afghanistan, the World Bank introduced a performance-based payment for NGOs. Through a competitive process, NGOs are contracted to provide a basic package of services to people living in an entire province. In this system, the winning NGOs receive a contract that pays them the budget they proposed plus the opportunity to earn up to an additional 10 percent if performance targets are reached. Overall performance is better in these World Bank provinces, leading other donors to consider PBF.

While briefing on the performance-based financial initiatives in the health sector in Bangladesh, Dr. Rob referred to the incentive provided in three programs.

- Family planning program, in promoting long-acting and permanent methods, provides incentives to providers and clients.

- Demand-side financing program for maternal health allows incentive payment on case basis to providers and pregnant women.

- Pay-for-performance program provides incentive to the institution for achieving predetermined performance targets of $\mathrm{MNCH}$ services.

\section{Performance-based financing has both benefits and limitations}

Dr. Rob mentioned several benefits and potential unintended effects of performance-based financing. He stated that performance-based financing is effective if collective model of motivation is emphasized rather than individual. Benefits of performance-based financing are: 
- Motivate changes in behavior

- Encourage staff to work in remote areas

- Promote hard work, innovation, and results

- Bring desired improvements in quality and performance

- Ensure greater accountability, i.e., improve the way health facilities respond to users, and

- Empower community or external entity to provide feedback on the quality of services.

Potential unintended effects of pay-for-performance include:

- More focus on aspects of clinical performance that are measured and neglect of unmeasured areas

- Diminishing professional motivation as a key attribute of quality health care

- More cost per service - rewarding providers who meet or exceed the target threshold, and

- Misreporting, gaming or fraud.

\section{Performance-based financing is a short-term solution}

Dr. Rob stated that PBF is just one promising and innovative strategy to tackle issues related to service use and provider performance. He added that performance-based financing makes a significant difference in a context where salaries remain inadequate. However, he cautioned that performance-based schemes are neither a cure for all problems of health systems nor a substitute for investments in health facilities. Performance-based financing is a short-term mechanism to expedite health results. 


\section{PERFORMANCE-BASED FINANCING IN BANGLADESH: POLICIES AND EXPERIENCES}

To ensure equity in access to health services in rural areas, the Government of Bangladesh has undertaken several initiatives, which include demand-side financing for maternal health services (implemented in 53 upazilas) and pay-for-performance for addressing low use of facilities for maternal, newborn and child health services (pilot tested in 3 districts). In addition, the MOHFW is developing health care financing strategy, which will incorporate the ways to mobilize sufficient resources and effectively manage them to serve the needs of the poor. The discussion that follows will highlight on these three initiatives.

\section{Demand-side financing scheme: Design, experiences, challenges and way forward}

Dr. Azizul Alim, Deputy Program Manager, DGHS, MOHFW described how demand-side financing (DSF) is designed, how it works and lessons from this program.

In Bangladesh, one of the limitations of the health sector is the low use of facilities for maternal health services in rural areas, which is influenced primarily by demand-side barriers. One of the main determinants of low utilization of professional maternity care is related to cost. The government health facilities provide free antenatal care (ANC), delivery and postnatal care (PNC) services, but the cost of medicines in the case of complications is not fully covered under the current service delivery system. In addition, transportation cost is not covered. In order to provide financial support to poor women for receiving safe delivery services in rural areas, the DGHS is implementing DSF program for maternal health care in several upazilas of the country. The overarching goal of DSF is to contribute to achieve MDG 5 by reducing maternal mortality.

DSF pilot project was launched in 2004, and became functional in two upazilas in 2006. Currently the program is implemented in 53 upazilas. Selection of upazilas depends on several criteria: availability of comprehensive emergency obstetric care facility; presence of skilled birth attendant program; poverty level; literacy rate; geographical location; and scope of public-private partnership. DSF is primarily implemented at Upazila Health Complexes, with referral to Maternal and Child Welfare Center or District Hospital. In selected upazilas, some union-level health facilities are included in the DSF program. Financing of the program is primarily channeled through "pool fund" administered by the World Bank where WHO, UKAid and UNFPA are the major donors. WHO is providing technical assistance through upazilla-based DSF organizers and the DSF cell in DGHS.

Poor pregnant women are the primary beneficiaries of the DSF program. The criteria used in the DSF program to select poor women include: 
- First or second pregnancy

- Resident in the respective upazila

- Functionally landless i.e. owning less than 0.15 acre of land

- Extremely low or irregular income (households earning less than Tk. 2500 per month), and

- Lack of productive assets.

Both demand and supply sides are financially benefited through the DSF program. At the demand side, poor pregnant women receive financial assistance for transportation cost to receive service from facility or for referral. In addition, they get Tk. 2000 for purchasing nutritious food if deliveries are conducted at the facility or by skilled birth attendant. Similarly, newborns of those women are provided a gift box costing no more than Tk. 500 .

At the supply side, service providers receive case-based incentive with a variation across public and private sectors. Designated providers are reimbursed upon verification of services. Public-sector providers are awarded 50 percent of total allocated cost against the service as incentive while the remaining is kept as seed fund for improving the facility, including drugs and infrastructure. On the other hand, private-sector providers receive 100 percent of total allocated cost.

Services for which providers are eligible to receive incentive include: registration, blood test (prenatal, twice), urine test (twice), antenatal check-up (three times), safe delivery (normal delivery), postnatal check-up, services for complications, manual removal of placenta, dilation and curettage, vacuum/forceps delivery, management of eclampsia, and cesarean section.

Amount of incentive varies according to the types of services. For example, total performance reward for cesarean section delivery is Tk. 6000 (US\$ 83) ${ }^{4}$, where public-sector providers receive Tk. 3000 as cash incentive, which is distributed among its 8-member team following predetermined benefit ratios. More precisely, surgeon gets Tk. 1100, anesthetist Tk. 600, operation assistant Tk. 500, two senior staff nurses Tk. 500, aya Tk. 100, ward boy Tk. 100, and cleaner Tk. 100. DSF program cost per mother is estimated at Tk. 3956 (US\$ 55), which includes both service cost and non-service cost. Under the payment mechanism, Upazila Heath and Family Planning Officer receives DSF fund directly from the Director, Primary Health Care, DGHS in a bank account and withdraws the cash for both provider incentive and client payment.

Six committees have been formed at different levels to implement the DSF scheme.

- National DSF Committee (Health Minister as Chair).

- DSF Program Implementation Committee (Health Secretary as Chair).

- DSF Technical Sub Committee (Director General of DGHS as Chair).

- District Designation Body (Civil Surgeon as Chair).

\footnotetext{
${ }^{4} 1 \mathrm{USD}=72 \mathrm{Taka}$
} 
- Upazila DSF Committee (Upazila Nirbahi Officer as Chair).

- Union DSF Committee (Union Parishad Chairman as Chair).

Dr. Alim noted that an economic evaluation of the DSF program was carried out in 2010. Findings suggest that DSF program is having positive effect on the utilization of maternal health services, as deliveries by skilled health personnel have significantly increased. Reduction of out-of-pocket expenditures is worth noting too.

Dr. Alim mentioned several limitations of the DSF program, which had been identified through the evaluation. There are some operational issues to be addressed: delay of fund release; delay in disbursement of cash incentive and gift box; insufficient monitoring; staff mix (obstetrician and anesthesiologist pair); procurement; and infrastructure. Moreover, eligibility is not properly followed to identify poor pregnant women. There is limited involvement of non-public providers, which implies the need to ensure multi-sectoral collaboration between the government, NGO, privatesector providers.

\section{P4P model: Context, design, experiences, challenges and way forward}

Ms. Laila Rahman, Senior Program Officer, Population Council and Dr. Riad Mahmud, Health Officer, UNICEF/Bangladesh jointly presented the P4P project. Dr. Mahmud discussed the context of introducing P4P in the health sector in Bangladesh while Ms. Rahman elaborated on its design, experiences, challenges and way forward.

\section{The context}

At the outset, Dr. Riad Mahmud provided the background for undertaking the P4P project. Dr. Mahmud described the current maternal, newborn and child health status in Bangladesh. He highlighted several supply-side challenges in the health sector, such as human resources, quality of care, and logistics and supplies. Despite comprehensive infrastructure, health system in Bangladesh faces a critical challenge on human resources. He stated that heath system was not able to provide quality services due to sub-optimal performance of providers, which was also compounded by the lack of required supplies and logistics.

Dr. Mahmud mentioned several aspects of human resource challenges that exist in the health system of Bangladesh.

- Poor working environment for providers.

- Frequent changes at the managerial level.

- Increased work load (e.g. 100-bed District Hospital run by 50-bed human resources).

- Low remuneration and lack of performance-based incentive.

- Only one pair of obstetrician and anesthesiologist to ensure 24-hour emergency obstetric care (EmOC). 
- Working of gynecology/obstetrics consultant of Upazila Health Complex in Medical College Hospital under deputation.

- Perceived lack of confidence of EmOC-trained doctors, especially for six-month training recipients.

- Tenure of EmOC-trained doctors in rural Upazila Health Complexes not linked to career pathway (e.g. Bangladesh College of Physicians and Surgeons does not recognize it as training).

Dr. Mahmud mentioned that current UNICEF activities have been directed towards improving maternal, newborn and child health. The government in collaboration with UNICEF and NGO partners has been implementing two $\mathrm{MNCH} / \mathrm{MNH}$ projects in eight districts, where UNICEF provides technical assistance in enhancing capacity of providers and strengthening facilities, and BRAC/CARE increases demand for services and strengthens capacity of community-based providers. Dr. Mahmud mentioned that shortage of skilled human resources for delivering quality $\mathrm{MNCH}$ services is common in these project areas, which prompted the introduction of $\mathrm{P} 4 \mathrm{P}$ for motivating service providers to improve the quantity as well as the quality of maternal, newborn and child health services in the existing $\mathrm{MNCH}$ and $\mathrm{MNH}$ projects.

\section{P4P project}

To begin with, Ms. Laila Rahman elaborated on the P4P model development. Ms. Rahman stated that upon request from UNICEF, the Council developed a model to introduce P4P for MNCH services in the GOB-UN's ongoing $\mathrm{MNCH}$ and $\mathrm{MNH}$ projects. The study utilized existing $\mathrm{MNCH} / \mathrm{MNH}$ project intervention components and introduced "pay-for-performance" model, i.e., paying providers a financial reward for achieving predetermined performance targets in terms of increased quantity as well as quality of services delivered.

\section{Prerequisites}

Ms. Rahman described the process of developing an implementable P4P model, which was the first and foremost task of P4P pilot study. To develop the P4P model, policy workshops (1 national and 4 district level) with both national and local level program managers and service providers were organized. Similarly, four workshops were held with faculty from Medical Colleges, representatives from Obstetrical and Gynecological Society of Bangladesh (OGSB), managers from District Hospitals and Upazila Health Complexes and consultants from District Hospitals. Based on stakeholder analysis (workshop findings), a P4P model that included five guidelines was developed. The mechanism for implementing and monitoring P4P initiatives into the government health system is delineated in those guidelines.

Ms. Rahman noted that three prerequisites were ensured before introducing P4P. The first prerequisite for implementing $\mathrm{P} 4 \mathrm{P}$ approach was that health facilities remain functional with necessary structure, supplies and trained providers. The second prerequisite was to have an inbuilt 'quality assurance system' for accrediting the facilities that provide minimum acceptable level of 
$\mathrm{MNCH}$ care and ensuring routine measurement of performance. Establishing an incentive payment mechanism was the last condition to meet before introducing P4P into the existing $\mathrm{MNCH} / \mathrm{MNH}$ projects.

\section{Research design}

Ms. Rahman briefly discussed the project objectives. She stated the long-term goal of the project as reducing maternal, neonatal and under-five child mortality and morbidity. The immediate objective of the study was to test and compare two service delivery models to increase the utilization of maternal and neonatal care services, and under-five children's critical health care services from the facilities.

Regarding study location, Ms. Rahman stated that the P4P project was implemented in three districts. She clarified that as the study was nested within existing $\mathrm{MNCH} / \mathrm{MNH}$ projects, three districts were purposively selected for intervention. The selected districts were Jamalpur, Gaibandha and Kurigram. From each district, one District Hospital and three Upazila Health Complexes (UHCs) received the interventions. Among three intervention UHCs, one UHC with comprehensive emergency obstetric care and two UHCs with basic emergency obstetric care were selected. A total of 12 units from 3 districts were exposed to interventions. Thakurgaon, an MNH intervention district, was selected as the control site. The experimental groups received the program interventions for 14 months.

While discussing study design, Ms. Rahman said that two strategies were tested across study sites. The first strategy introduced a P4P scheme for motivating service providers to improve the quantity as well as the quality of services and enabled poor pregnant women and mothers of newborns and under-five children to access services by reducing their out-of-pocket costs. The second strategy constituted a P4P scheme for providers only. Thus, the first strategy provided cash incentive for both providers and poor clients while the second strategy provided cash incentive for providers only. Gaibandha and Kurigram districts were randomly assigned to strategy 1 while Jamalpur district for strategy 2.

\section{P4P Model}

Ms. Rahman described the process to assess facility performance and to pay incentives to service providers and

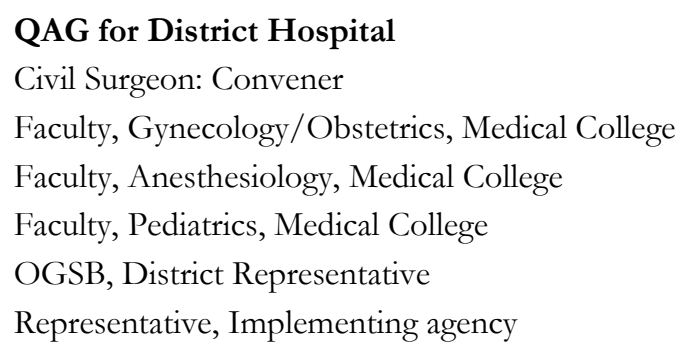


recipients. In particular, she highlighted the quality assurance, incentive recipients, payment rules, and project management.

Establishment of quality assurance mechanism. Quality assurance groups (QAGs) were formed within each district, consisting of specialists from nearby Medical College Hospital or District Hospital and professional body, with the purpose to provide systematic visits to health facilities to accredit and assess. QAGs visited the facilities quarterly, graded the facilities and provided supportive feedback to the providers to improve maternal, newborn and child health services.

Within the facility, the MNCH team was divided into smaller teams or sub-teams to provide specific services, as an internal quality assurance mechanism. Quality assurance teams (QATs) were formed for the following units: emergency room, labor room, operation theater, autoclave room, female ward, child ward or newborn care corner, pharmacy, store, laboratory, antenatal and postnatal care corner, and family planning corner. For each of the QATs, a leader was identified and given responsibility to coordinate the activities of the respective unit. QATs monitored and reviewed service performance fortnightly and ensured coordination between team members.

Formation of P4P Committees. Institutionbased P4P Committees were formed to implement, oversee and facilitate the P4P project activities. The committee members met once a month to review the performance of the facility, and took appropriate measures, as necessary. As part of the financial management under $\mathrm{P} 4 \mathrm{P}$ project, the committees were mandated to receive the incentive and the "drugs, consumables and maintenance" fund in the P4P Committee's bank account, keep appropriate records, and approve of incentive distribution to the beneficiaries.

Incentive recipients. Incentives were

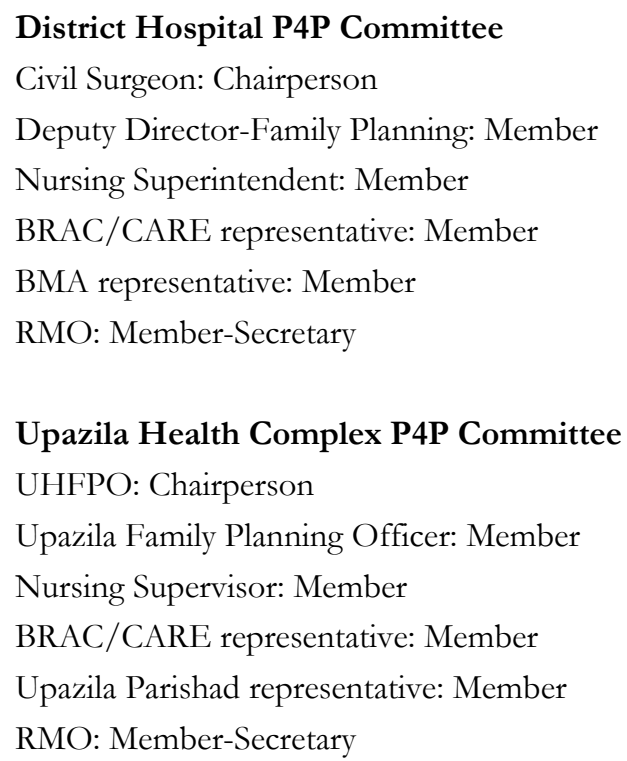
provided to motivate service providers at the institution level. Since the main thrust of introducing P4P approach was to increase institutional deliveries, direct and indirect providers related to pregnancy, neonatal care and under five children's services, and management, administrative and support staff were eligible to receive incentive if the performance targets were achieved or exceeded.

Performance targets. Quarterly targets for $\mathrm{MNCH}$ services were set for the institution as a whole, which took into account both quantity and quality of services. Two levels of quantitative performance targets based on the benchmark were set to pay incentives to a health facility. Amount of incentive of a facility varied according to its level of performance improvement - higher incentive payment for higher performance improvement. Incentives payable to providers and staff were 
calculated on the basis of level of efforts; for instance managers and direct service providers received full incentive while indirect service providers and administrative staff received half incentive. QAG, in consultation with respective facility-based P4P committee, determined performance targets, performance achievements, and eligibility for incentive. Performance of a facility was measured using service statistics and QAG visit reports.

Reimbursement mechanism. A systematic financial reimbursement mechanism was employed for calculating, disbursing and verifying incentives. At the end of each quarter, performance was measured and the level of the incentives was determined. Incentive payment related to performance was determined by the QAG members, and reimbursed by the project implementing agency. The project staff calculated the amount of incentives payable to the service providers by using the QAG visit report and analyzing service statistics. Service providers received money quarterly through bank transfer for achieving the facility-based performance targets. Meanwhile, the disbursement of fund was validated by an audit firm.

Reaching poor clients. Poor pregnant women in the project areas in Gaibandha and Kurigram districts received financial assistance to meet costs of transportation, medicines and other incidentals for pregnancy and delivery related services. Newborns and under-five children of poor mothers received similar financial assistance too. Coupons were provided to cover transportation, medicines, and incidental costs relating to unforeseen expenses women, newborns, and under-five children incurred while staying a facility. Medicines were provided on receipt of a medicine coupon, while cash was provided to beneficiaries to meet transportation and incidental costs after receiving services.

\section{Findings from P4P project}

Ms. Rahman highlighted several key findings of the P4P pilot project. She said that the P4P model contributed in improving the quality of care while increasing the quantity of services. Assessment of the performance indicates that in 93 percent of cases, facilities received incentives based on achieving both the quantity and quality targets. $\mathrm{MNCH}$ service volume increased remarkably due to the P4P interventions. For example, each District Hospital doubled the performance in institutional delivery while each Upazila Health Complex tripled that performance.

\section{P4P: Key lessons learned}

- Facilities respond to the performance targets in terms of increasing quantity and quality of $\mathrm{MNCH}$ care.

- Providers are motivated with P4P incentives.

- Nurses, indirect providers and support staff perceive more financial benefits than doctors/consultants.

- QAG tool empowers the QAG members to measure performance and provide feedback.

- Mentoring through the QAGs is effective but does not replace the need for formal training.

- Accountability of the providers can be increased through internal unit-based quality assurance teams.

In terms of quality, facilities that have achieved performance targets used partograph in all deliveries and improved the readiness of labor room, emergency room, female ward, pediatric ward, laboratory and store. Un-sterile and sterile areas of autoclave room were marked, with a trained person in- 
charge. The facilities also introduced antenatal care and postnatal care corner, breastfeeding corner, post-operative room, and washroom adjacent to labor room. In all facilities, sick newborn care unit was separated. Suggestion box was installed for clients to provide feedback on the improvement of services.

Ms. Rahman also provided some cost information related to the provider incentive and institutional delivery. Average quarterly provider incentive cost per District Hospital was estimated at Tk.

740,666 (equivalent to US\$10,287) which was distributed among 63 staff. Direct providers received the majority of the incentives (67 percent) followed by support staff (12 percent). Managers got only eight percent of the total incentive allocated to the institution.

Each Upazila Health Complex received Tk. 436,009 (equivalent to US\$ 6,056) as provider incentive per quarter, which was distributed among its 44 staff. Like District Hospital, nearly similar distribution of incentives was observed for the Upazila Health Complexes. Of the total incentive allocated to the institution, direct providers received the majority of the incentives (59 percent) followed by support staff (18 percent) and managers ( 9 percent).

Average rate of each institutional delivery stood at Tk. 4,556 (US\$ 63) which varied between districts mainly due to staff size of facilities and number of deliveries. In Gaibandha, US\$ 53 was required as provider incentive for each institutional delivery while it was US\$ 65 and 67 for Kurigram and Jamalpur respectively.

\section{Way forward}

Ms. Rahman mentioned several challenges that were encountered during the implementation of P4P project. The challenges were:

- Placement of key human resources was prerequisite, but not met.

- Some facilities were chronically constrained by vacancy in key human resource positions.

- Lack of training/refresher training hindered the quality improvement.

- Inadequate utilization of the project fund limited the capacity of unit-based quality assurance teams to bring the desired change.

Ms. Rahman put forward some recommendations for improving the P4P model:

- Key human resources are to be placed and provided with training/refresher training.

- An automated QAG tool could be more effective.

- Health management information system needs to be automated.

- Larger group of QAG members or an institution needs to be aligned, trained, certified and engaged.

- Institutional targets based on past performance should not be used for the facilities that 
historically performed at low level, otherwise it will increase the cost of delivery per facility.

- It is necessary to integrate audit on service volume and client perspective with performance measurement.

- Minimum quantity of institutional delivery needs to be defined in order to ensure sustainability.

Ms. Rahman justified the necessity for continuing provider incentive to improve the MNCH services by illustrating the positive results of $\mathrm{P} 4 \mathrm{P}$ pilot project. She concluded her presentation with the hope that P4P model's "quality assurance system" could be adapted into the DSF scheme for facility strengthening and employing standardization in quantitative and qualitative achievements.

\section{Health care financing strategy: Process and progress}

Mr. Prasanta Bhushan Barua, Joint Chief, Health Economics Unit, MOHFW described the process of development of a "Health Care Financing Strategy", focusing on the context, approach and progress. Mr. Barua was interested to learn from the workshop how performance-based financing can be incorporated as one of the strategies for health care financing.

Strategic plan documents of HPNSDP 2011-16 necessitate the development of a health care financing strategy. Mr. Barua informed that the Government of Bangladesh recognizes that it faces a challenge in mobilizing sufficient resources and effectively managing them to serve the needs to the poor. With an increasing share of health expenditures being financed by household in the form of out-of-pocket payments and the prospect of declining contributions from development partners in future, the MOHFW made an attempt to review and reform the way in which the sector is financed.

To begin with, the MOHFW formed a "Health Financing Resource Group" comprised of representatives of government, development partners, academics, and researchers, who will lead the process of developing the strategy. The MOHFW has adopted a systematic process to develop health care financing strategy. Mr. Barua discussed the steps of developing the strategy. Broadly, the activities are divided into five steps or stages:

- Set goals and objectives

- Identify and review potential strategies

- Draft the health care financing strategy document

- Conduct stakeholder consultations and finalize strategy document, and

- Approval of the strategy.

Mr. Barua shared a draft outline of the health care financing strategy. He stated the vision of the proposed strategy as to "...support universal coverage of health care focusing on equity, efficiency and resource mobilization.” The strategy document will include five broad sections: 
- Rationale/situation analysis

- Health care financing vision, goals and objectives

- Health care financing strategies (with examples)

- Implementation framework, and

- Monitoring and evaluation framework.

Mr. Barua informed that the MOHFW is currently at the stage to identify and review potential strategies. He expected to share the first draft of the strategy document within March 2012. Upon undertaking stakeholder consultations and revisions, final draft will be ready for approval by May 2012. To conclude, he appreciated the timeliness and appropriateness of the workshop on performance-based financing, which provided a useful platform to understand the strengths, limitations and challenges of DSF and P4P schemes, and to identify short-term and medium-term strategies/recommendations for DSF and P4P as part of the health care financing policies. 


\section{DEFINING FUTURE PERFORMANCE-BASED \\ FINANCING MODEL: FINDINGS FROM \\ GROUP WORK}

The second day of the workshop was primarily dedicated to group discussion. Two groups were formed and assigned specific performance-based financing programs (DSF and P4P) to examine their status, identify issues and challenges to implement these programs, and compile understanding on how to address those issues.

Each of the two groups consisted of selected group members and a pre-designated group leader and a facilitator. Members of the respective group selected a "presenter" for sharing the findings of "group work". Discussion of the group work centered on seven broad areas.

- Implementation facility

- Incentive beneficiaries (supply side and demand side)

- Performance measurement criteria and authority

- Quality assurance system

- Level of payment

- Incentive payment mechanism, and

- Challenges.

The group work was designed in a way that understanding of the issues and limitations of DSF and P4P schemes was improved and that two sets of recommendations were drafted:

- Incorporation of the quality of care framework and modification of financing mechanism in DSF scheme.

- Modification of the institution-based P4P model.

\section{Group work findings}

\section{a) Incorporation of quality of care framework and modification of financing mechanism in DSF scheme \\ Group A discussed on the feasibility of incorporating quality of care framework and modifying financing mechanism of the DSF scheme. The group was consisted of 32 members. Among them Dr. Saleh Mohammad Rafique, former Director, Primary Health Care, DGHS was the team leader and Dr. S.M. Shahida, Assistant Professor, Gynecology/Obstetrics, Mymensingh Medical College was the presenter. Mr. Md. Moshiur Rahman, Program Officer, Population Council facilitated the discussion.}


DSF scheme is primarily implemented at Upazila Health Complexes, with referral to Maternal and Child Welfare Center or District Hospital. DSF is the first attempt in the health sector that provides financial support to poor women for receiving safe delivery services from facilities. Supply side is also financially benefited through the DSF program where service providers receive incentive for obstetric care services. In selected upazilas, union-level health facilities are included in the DSF program. Group A suggested the inclusion of all union Health and Family Welfare Centers $(\mathrm{HFWCs})^{5}$ of a upazila into the DSF scheme. Dr. Shahida, the presenter from Group A, identified non-availability of delivery services at HFWCs as a key challenge to ensure access to safe delivery services at the community level. HFWCs need to be strengthened to provide normal delivery services and to promote referral from community to upazila and district level hospitals.

Under the DSF scheme, incentives are given to facility-based providers while field-level functionaries (health and family planning fieldworkers and their supervisors) are not entitled as beneficiary. At the demand side, DSF aims to provide financial assistance to only pregnant women for receiving obstetric care services. Dr. Shahida, in her presentation, urged policymakers to include health and family planning fieldworkers and their supervisors as beneficiary at the supply side and newborns at the demand side. Most importantly, whole institution approach should be adopted, i.e., all units of a facility should be brought under the umbrella of performance-based financing.

Only quantity of services is considered to measure the performance of providers under the DSF scheme. Dr. Shahida noted that DSF offers incentives based on number of services provided to the voucher clients, for which non-voucher clients do not often get equal attention. She emphasized to have a defined performance measurement system in the DSF scheme, which should consider both quantity and quality of services.

Incentives are provided monthly, calculating the total number of services provided to poor pregnant women, for which there is no external body to assess the performance. Dr. Shahida referred to the P4P project in outlining the performance measurement system for the DSF scheme. Like the P4P project, quality assurance group (QAG), consisting of experts from Medical College or District Hospital, should periodically measure the performance of a facility using a standard checklist.

The presenter elaborated on a "quality assurance system" for the DSF scheme. Group A suggested to adopt the P4P experiences of introducing both external and internal quality assurance system. For external quality assurance system, DSF scheme can form QAGs within each district, where faculty from Medical College will visit Upazila Health Complex with comprehensive emergency obstetric care and consultants from District Hospital for Upazila Health Complex with basic emergency obstetric care. Like the P4P project, QAGs may make quarterly visits to facilities, measure the performance of facilities and provide supportive feedback to the providers to improve maternal,

${ }^{5} \mathrm{HFWC}$ is the first level fixed-facility located at the union level (the lowest administrative unit covering about 30,000 population) which provides outdoor services from 8:00 am to 2:30 pm six days a week. 
newborn and child health services. As part of internal quality assurance system, unit-based quality assurance teams, same as $\mathrm{P} 4 \mathrm{P}$, can be formed to monitor and review performance routinely as well as to supervise the quality within facility.

At present, Upazila DSF Committee, which is headed by Upazila Nirbahi Officer, has four members from the Upazila Health Complex and more than five non-facility members, but consultants of the respective facility are not included as members. For better functioning of DSF, Group A suggested to include Obstetrics and Anesthesiology Consultants of respective facility in the Upazila DSF Committee as members.

Group A identified several problems in the payment level and reimbursement mechanism of the DSF scheme. Under the DSF scheme, only providers receive incentives while managers and administrative staff are not covered as beneficiary. Other problems with payment level which need immediate attention are:

- Increase amount of benefits for both providers and service recipients.

- Introduce referral fees for community-based skilled birth attendant (CSBA) to identify highrisk pregnant women and to promote institutional delivery.

- Provide financial benefits to CSBA for performing delivery at home.

- Reduce the gap in incentive amount between surgeon and anesthesiologist.

Regarding incentive payment mechanism, Group A suggested adopting P4P approach in providing incentives to the facility. Generally, Upazila Heath and Family Planning Officer receives DSF fund directly from the Director, Primary Health Care, DGHS in a bank account and withdraws the cash for both provider incentive and client payment. At the workshop, payment mechanism used in the P4P project was strongly recommended for the DSF program - a mixed system with bank transfer for provider payment and cash payment for clients. Financial management needs to be remained with the government. Performance should be measured quarterly replacing the current monthly measurement system.

On the basis of discussions among members at the group session, the presenter pointed out several challenges to strengthen DSF program, which included:

- Address shortage of human resources, especially obstetrician-anesthesiologist pair.

- Ensure functional ambulance facility.

- Develop infrastructure.

- Change attitudes of service providers.

- Modify human resource management strategy. 
Dr. Shahida concluded the presentation on behalf of Group A by reiterating that since the DSF scheme focuses on the quantity of services, the quality of care approach to improve MNCH services tested under the P4P scheme may have the potential to enrich the DSF scheme, and the improvement of the monitoring of health service delivery in Bangladesh.

\section{b) Modification of the institution-based P4P model}

Group B presented the P4P model and the group discussion was led by Dr. A.B.M. Jahangir Alam, former Director, Primary Health Care, DGHS. The group was consisted of 34 members, and identified some operational problems of P4P pilot project and put forward suggestions for modifying the existing model. Dr. A.B.M. Jahangir Alam was the team leader and Dr. Md. Feroze Khan, Upazila Health and Family Planning Officer, Islampur Upazila Health Complex, Jamalpur was the presenter. Mr. Md. Noorunnabi Talukder, Program Officer, Population Council, facilitated the discussion.

Dr. Md. Feroze Khan, the presenter from Group B, mentioned the absence of a unified strategy within the district health system since P4P approach was piloted at district and selected upazila hospitals. It was strongly suggested to adopt a whole district approach (all upazilas with both upazila- and union-level facilities) in phased manner, with strengthened referral from union to upazila to district. Inclusion of Mother and Child Welfare Centers (MCWCs) and HFWCs is another recommendation for

"District Hospital is the referral hospital, which receives clients from all its upazilas. More clients are coming to P4P upazila hospitals as they get financial assistance for referral services. Referral system will work better if all Uparila Health Complexes of a district receive P4P intervention. It should be a whole district approach."

- Dr. Nazrul Islam, RMO, Kurigram District Hospital modifying P4P approach.

P4P is noted for providing incentives to motivate service providers at the institution level covering management, service, administrative and support staff. $\mathrm{P} 4 \mathrm{P}$ project implemented two strategies in piloting the approach - one strategy tested incentives to both providers and clients while the other for service providers alone. Group B recommended not to implement two strategies in a single program, but to undertake a combined approach, i.e., providing financial assistance to poor clients along with rewarding service providers with incentive.

Services for which quantitative targets are set for the facility to receive incentive include: antenatal check-up, normal delivery, postnatal check-up, cesarean section delivery, and management of complications for newborn and under-five children. But, health facilities exert tremendous efforts to manage several deadly complications related to pregnancy and delivery which are not included as quantitative targets. By identifying such inadequacy of quantitative performance indicators, Dr. Khan emphasized the need to include new indicators, e.g., eclampsia

"We need manpower first then incentive. It is not possible to continue P4P program if we do not have necessary human resources, including doctors, nurses, ward boy, aya and cleaner. Developing specialists along with creating necessary post is a prerequisite for continuing such incentive program."

- Dr. Md. Shahidul Huq, Civil Surgeon, Gaibandha 
management, removal of retained placenta, management of ante-partum and post-partum hemorrhage, dilation and curettage, and post-abortion care.

The major limitation of the P4P model as identified by Group B is that family planning unit of a facility does not have separate targets and responsibilities. They automatically become eligible to receive the incentive once the health unit achieves the performance targets, which is a great source of de-motivation for some providers. Dr. Khan informed that the necessity to introduce separate targets for the family planning unit was repeatedly highlighted during the group work.

P4P project management committee is restricted to six members of which four members are from the respective facility, but does not have any consultants as its members. The presenter recommended to increase the size of $\mathrm{P} 4 \mathrm{P}$ committee by including Obstetrics, Pediatrics, and Anesthesiology Consultants of the respective facility (if any) as its members.

As part of external quality assurance system, Group B was supportive of continuing with the existing system, where faculty from Medical College visit District Hospital and Upazila Health Complex with comprehensive emergency obstetric care and consultants from District Hospital visit Upazila Health Complex with basic emergency obstetric care. For implementing quality assurance system at all upazilas, availability of experts from tertiary level hospitals will be a challenge. In the interim period, a QAG may be opted for certain number of facilities. Besides, quality supervision from district to upazila was suggested. Group B was in favor of increasing the existing rate of honorarium of QAG members for visiting health facilities by considering the distance and the opportunity cost.

P4P pioneered in implementing internal quality supervision by forming "quality assurance teams" for different service units within the facility. Dr. Khan commended it as a positive development since QAT meetings facilitate and ensure coordination and cooperation among the team members. Group B suggested of holding frequent meetings for QATs, e.g., weekly QAT meeting, as a way to sustain teamwork and ensure accountability. There should be some refreshment for QAT meeting.

Group B illustrated that within a facility, not all providers perform equally as the eligibility of a facility to receive performance bonus largely depends on direct providers, particularly the obstetric unit. Despite weak functioning and unsatisfactory performance of other units, performance of obstetric unit helps the facility to get incentives. In this connection, Dr. Khan mentioned the recommendations emerged from the group work:

- More incentives should be given to direct providers, particularly outstanding performers.

- It is necessary to empower facility managers so that they can reward the outstanding performer, and take measure for excluding non-performer from the incentive package.

- There should be regular coordination of services and an internal quality supervision system for improving the performance of all service units within the facility. 
On the basis of the discussions among members at the group session, Dr. Khan pointed out several recommendations to strengthen P4P model:

- Continue essential obstetric care training.

- Continue pediatric training.

- Create more number of anesthesiologists.

- Empower facility managers to undertake local recruitment.

- Provide ultrasonogram with required staff to Upazila Health Complex.

- Ensure no transfer or deputation during project period, without appropriate substitute.

- Revise and abridge the quality assurance checklist.

- Orient internal service units on quality assessment indicators. 


\section{PROSPECTS AND CHALLENGES OF PERFORMANCE- BASED FINANCING: STAKEHOLDERS' VIEWS}

In the discussion session, both present and former Directors of Primary Health Care, DGHS shared their experiences and provided insightful comments. Several experts from government and nongovernment sectors provided thoughtful inputs on the subject. Finally, the Secretary of MOHFW provided useful directions towards implementing performance-based financing for maternal and newborn care services in Bangladesh.

\section{Establishing quality assurance system and using local resources}

Dr. Saleh Mohammad Rafique, former Director, Primary Health Care, DGHS provided two specific suggestions regarding DSF: establishing a quality assurance system, and allowing the facility to use locally generated fund.

In Dr. Rafique's opinion, DSF is functioning well and contributing to the progress in the reduction of maternal deaths and therefore it should continue. He identified the absence of a defined quality assurance system as one of the limitations of DSF scheme. In addition, DSF does not promote any target-based performance. Dr. Rafique commended the $\mathrm{P} 4 \mathrm{P}$ approach as it provided incentive to the whole institution not to individual providers. To improve the functioning of DSF scheme, Dr.

"It may not be feasible to form quality assurance group with the experts from medical college hospital for monitoring all upazilas. It is necessary to know how many experts are available for periodic supervision of Upazila Heath Complexes. One quality assurance group may visit multiple facilities. Alternatively, there can be quality supervision form Medical College to District Hospital and from District Hospital to Upazila Heath Complex." - Dr. A.K.M. Sadiqul Azam, Assistant Professor, Mymensingh Medical College Rafique advised to adopt the lessons of P4P project which successfully implemented both targetbased performance and systematic quality assurance mechanism.

Incentive improves the provider performance and the quality of health services, but it is not a permanent mechanism. Dr. Rafique wanted to know how long such boost mechanism would continue. The most pressing challenge for future of DSF will be securing sustainable financing. Dr. Rafique urged the policymakers to devise strategies of how to sustain the DSF program once the donor assistance is withdrawn.

Dr. Rafique strongly advocated for introducing "user fees" in all Upazila Health Complexes in an attempt to use private funds to address resource gap, i.e., to supplement government budgetary resources. He, however, noted that in a limited scale, Upazila Health Complexes started to charge user fees for selected services, like admission fee, pathological fee and ambulance charge. Nevertheless, centralized financial control has undermined the effectiveness of user fee. Local hospitals should be allowed to keep the user fees that they collect rather than return them to the state treasury. The benefits of such arrangement will be manifold: hospitals can re-invest the fund in its smooth functioning, emergency repair and maintenance, purchasing fuel for ambulance, 
procuring essential equipment, drugs and other medical supplies (if needed), and subsidizing or exempting fees for poor patients. Dr. Rafique called for immediate attention of policymakers towards empowering the local managers to retain user fees for sustainable improvement of quality of services.

\section{Implementing a modified program}

Dr. A.B.M. Jahangir Alam, former Director, Primary Health Care, DGHS attached greater importance to demand-side financing along with providing incentive to the institution. He urged policymakers to adopt good practices of both P4P and DSF programs.

Dr. Alam expressed his concerns on the persistent underutilization of health facilities in rural areas. Health-system service delivery in rural areas of Bangladesh faces several challenges on both demand and supply side. To increase the use of facilities, it is necessary to address four issues: poor people's inability or lack of affordability for defraying the cost of transportation and medicine; lack of awareness on the availability of free services at government hospitals; geographical access in hard-toreach areas; and attitude of providers or lack of motivation among providers. In this connection, Dr. Alam cited examples of DSF scheme in overcoming those barriers to increase the use of facilities.

On the other hand, several features of the P4P approach attracted Dr. Alam's attention.

- Dr. Alam admired the design of P4P in providing incentive to both direct and indirect providers as well as management, administrative and support staff. In $\mathrm{P} 4 \mathrm{P}$, incentives are given to motivate service providers at the institution level, instead at the individual level as practiced in DSF.

- There is a missed opportunity for the DSF scheme that providers working at the obstetric unit receive the incentive while service providers working at other units are not included as beneficiary. Dr. Alam advised to adopt the P4P's team approach in covering the whole institute.

"Under P4P project, both teamwork and rearrangement of available staff within the facility have been successfully adopted toward achieving performance targets, but not contributing to skills development. For instance, in a District Hospital, nurses have to work at different units by rotation, which leaves them with little opportunity to acquire specific skills. If nurses, ward boys and ayas are placed in a unit for long time, they will improve their skills while working under a fixed team."

- Dr. Md. Tazul Islam, Junior Consultant (Pediatrics), Jamalpur District Hospital

- At the demand side, DSF targets the poor pregnant women. Dr. Alam shared P4P example where neonate and under-five children's services are targeted in addition to the services for pregnant women, which can be utilized for the DSF scheme.

- In DSF, provider incentive is given on the basis of individual performance (quantity). Dr. Alam suggested the inclusion of quality as a measurement indicator in the DSF scheme. 
Dr. Alam was skeptic about introducing user fees as he considered poverty a key obstacle to mainstream such initiative. Poor families in rural areas should be given financial assistance in seeking health care.

P4P project implemented two strategies in piloting the approach - one strategy tested incentives to both providers and clients while the other for service providers alone. Dr. Alam noted that the exclusion of demand-side financing in one of the P4P districts is a barrier for poor clients to seek services from facilities. He strongly recommended providing financial assistance to poor clients. There should be a combined strategy in P4P project - incentive to both providers and poor clients. Dr. Alam concluded by stating that the government can adopt the good practices of both DSF and P4P programs and implement a modified program, not two nearly similar parallel programs.

\section{Views of experts}

Mr. Dhiraj Kumar Nath, former Advisor to the Caretaker Government of Bangladesh, was impressed with the speedy success of P4P study, which was reflected in the remarkable increase of the use of facilities for $\mathrm{MNCH}$ services. Due to the incentive system, providers are committed to work round-the-clock. Mr. Nath, however, questioned the sustainability of the program if cash rewards are withdrawn from the system. Even worse, the government does not have the ability to continue such cash incentive with its limited resources. As providers have developed the habit of getting cash incentives for their performance, it will be difficult to keep their performance continue once such benefits are withdrawn. Mr. Nath advised to find effective ways to reward better performers.

Mr. Nath proposed insurance mechanism as an alternative health financing system and advised Health Economics Unit of MOHFW to incorporate it in the health care financing strategy. Additionally, Health Economics Unit should attach appropriate attention to the sustainability of performance-based financing programs while developing the strategy.

Dr. Jahiruddin Ahmed, former Director, Maternal and Child Health, DGFP was not supportive of the idea to provide cash incentive for carrying out regular job responsibilities. He opined that providing incentive along with salary is a discriminative measure and such incentive is not at all sustainable. Nevertheless, Dr. Ahmed was in favor of running such program only in disadvantaged or hard-to-reach areas. He advised program managers to be farsighted in spending the resources available for the health sector. More emphasis should be given to improve the management capacity with systematic supervision and monitoring. Dr. Ahmed identified some risks associated with P4P initiative.

- Incentive may be counterproductive if required manpower, logistics and training are not in place. In such cases, money will not lead to willingness, rather morality may be waned.

- If P4P is implemented in certain upazilas of a district, other upazilas will not work what they are supposed to work. P4P can be systematically introduced throughout the whole district if there is enough money. 
Dr. Barkat-E-Khuda, Professor of Economics, University of Dhaka centered his discussion on some managerial issues. Professor Khuda was agreed with the practice of introducing incentive at the initial stage of a targeted health, population and nutrition program or for covering hard-to-reach populations. He opined that when a program is matured or relatively matured, such incentive should not be continued because it is not sustainable. He advised to consider three issues prior to implementing a program - access, quality and sustainability.

Professor Khuda identified a gap in the $\mathrm{P} 4 \mathrm{P}$ program, as there is no system of punishment for poor performers. He was not convinced with the idea of only rewarding better performers. For any incentive-based financing program, there should be two sides - better performers need to be rewarded and poor performers should be punished. Professor Khuda strongly recommended disincentives along with incentives.

Mr. Yann Derriennic, Principal Associate, Health Systems and Financing, Abt. Association/Health Systems 20/20, was encouraged by the positive results of both DSF and P4P experiences, but he was concerned with the transitory life of these effective programs. Mr. Derriennic urged the government to have a policy on the continuation of performance-based financing programs because such encouragement is required until certain level of institutional deliveries is reached. He opined that incentive should not only be for number of deliveries conducted, but improvement in quality also be reckoned for rewarding incentive.

In Mr. Derriennic's opinion, it will be more realistic if there are facility-specific performance targets to receive incentive payment. It should be local-level managers and their supervisors who will set the target, not necessarily the central management. Incentive programs will be benefited if such local-level target setting approach is employed, leaving aside the uneven competition between facilities.

Mr. Derriennic was optimistic of introducing quality assurance in DSF. But, scaling up from
"Both district and upazila managers should be empowered. Central management requires a long process to make posting at the Upazila Health Complex. Civil Surgeon needs to rearrange the workforce across Upazila Health Complexes, so that every Upazila Health Complex will have minimum required staff-mix. Upazila Health and Family Planning Officer should be empowered to undertake local recruitment and take corrective measure for non-performers." - Dr. Md. Feroze Khan, UHFPO, Islampur UHC, Jamalpur 9 to 53 upazilas will be extremely challenging, because of the constraints of human resources.

Performance-based financing can have positive results, but such programs should not continue forever, rather it should continue for reaching certain targets within a specific time and providing sufficient evidence to come up with long-term solution. Mr. Derriennic called for the attention of policymakers and program managers to adopt results from both DSF and P4P and advance with a revised model, which will require less money. 


\section{Speeches of special guests}

\section{Reforming the performance-based financing model for quality $\mathrm{MNCH}$ services}

Mr. Michel Saint-Lot, Deputy Representative, UNICEF appreciated the appropriateness of

performance-based financing workshop in the context of the government's commitment of ensuring quality health services to mothers and children. Bangladesh made an impressive progress in reducing child mortality and saving mother's life, however, the country is still facing challenges to meet its MDG commitment and to improve quality in health care services. Being optimistic, Mr. Saint-Lot referred to the effectiveness of P4P and DSF programs in addressing maternal, neonatal and child mortality and morbidity as well as ensuring human rights to health and to live.

Mr. Saint-Lot highlighted several systemic achievements of the P4P project, which has increased the use of maternal, newborn and child health care through providing performance-based incentives to service providers and subsidized coupon to poor women, newborn and under- 5 children. P4P project has achieved its first target by developing human resources model to improve MNCH services of a facility. Quality of care was the heart of the program, which is not currently addressed in the DSF scheme.

No doubt, DSF and P4P are two innovative approaches to find a way to reduce maternal mortality, but they are laden with a risk of sustainability. It is important to know how long such trial should continue, but certainly for a short period. Mr. Saint-Lot emphasized the need for cooperation between the government and development partners to find solutions towards the sustainability of performance-based financing programs, to keep up the momentum generated through those programs, and to identify strategies of where to implement such program.

Mr. Saint-Lot looked ahead for revised performance-based financing models, which will improve both quality and quantity of MNCH services especially among the poor in Bangladesh.

\section{Synchronizing maternal and child health services of health and family planning}

Dr. Mohammed Sharif, Director, Maternal and Child Health, DGFP stated that incentive attached to the acceptance of permanent and long-acting family planning methods contributed to the success of family planning program in 1980s. Dr. Sharif appreciated the recent initiative of health sector in introducing performance incentives for $\mathrm{MNCH}$ services. He, however, underlined the necessity of performance-based financial incentive for the broader health system, for which functional coordination of services at the field level between health and family panning directorates is a prerequisite.

Dr. Sharif noted that incentive brings positive outcomes even in the shortage of providers. At the upazila level, approximately half of the doctors against sanctioned posts are available in position in the family planning service delivery. Most of these medical officers are providing services in more than one upazila, prompted by an increase in provider incentive. 
Dr. Sharif expressed his interest to work with the health unit despite separate process of accountability for health and family planning directorates. It is necessary to synchronize maternal and child health activities of both health and family planning units so that there will be coordination within whole health system at the district level and below. Dr. Sharif stressed for a concerted action to make the P4P initiative successful.

\section{Journey towards sustainable financing}

Mr. Prasanta Bhushan Barua, Joint Chief, Health Economics Unit, MOHFW centered his discussion on identifying alternative health care financing mechanism. Mr. Barua also made a comparison between DSF and P4P programs implemented by the Government of Bangladesh. Both the programs have the same goal of achieving MDG of reducing maternal mortality, but differ in approaches.

Regarding duration of programs, DSF has been functioning for the last five years while P4P was tested for only one year. Mr. Barua noted that under the purview of DSF only poor pregnant women are covered. The good thing about the $\mathrm{P} 4 \mathrm{P}$ project is that it covers services for neonates and under-five children along with pregnant women. Providing incentives to service providers and management, administrative and support staff based on their level of efforts contributed to ensure teamwork as a way to improve outcomes, which is another unique characteristic of the P4P approach. DSF does not link provider performance with quality of service, where the quality issues remain unaddressed. Mr. Barua appreciated the quality assurance system implemented under P4P project.

Mr. Barua justified the need to implement alternative health care financing mechanism. In his opinion, performance-based financing is not an alternative approach to health financing, as it does not generate any revenue rather it requires a large investment. Mr. Barua underlined the necessity to introduce "user fee" at the government facilities. He referred to the successful experiment on user fee being implemented in Hyderabad, India where local managers are allowed to retain and use the fund generated through user fee. Seventy percent of this locally generated fund is used for strengthening the facility while the remaining amount is given as incentive to providers. Mr. Barua proposed "health insurance" as another approach on alternative health care financing.

Resource mobilization through community involvement can be an effective tool for sustaining a program. Mr. Barua expressed his concerns with the inactivity of health management committees from community to higher level despite the government having specific order for forming committees at different tiers of health-system service delivery. He was optimistic that if DSF and $\mathrm{P} 4 \mathrm{P}$ could reactivate those committees, the community might own those programs in future.

Mr. Barua was hopeful that a revised P4P model would be implemented in a broader scale. Whatever is attempted, sustainable financing remains the key challenge. 


\section{Speech of the chair}

Dr. Md. Shamsul Haque, Director, Primary Health Care, DGHS began his speech by justifying the necessity of two incentive-based programs. Dr. Haque described the context for starting such incentive programs. Generally, poor people are denied access to social services, including health care. At the demand side, inadequate perception of women on the necessity for institutional delivery, lack of awareness on the availability of free maternal health services at government hospitals and geographical barriers contribute to the low use of facilities for delivery care. At the supply side, there is the problem of absenteeism, and poor accountability of service providers. The government's inability to retain trained providers in rural areas is also apparent.

Started in 2006, DSF is the first attempt in the health sector that ensures affordable access to facilitybased maternal health services among poor pregnant women. A major limitation of DSF scheme is that individual providers are entitled to receive incentive while $\mathrm{P} 4 \mathrm{P}$ provides incentive to the whole institution, covering both direct and indirect providers as well as managers, administrative and support staff. Dr. Haque called for the reform of DSF and adopting the good practices from P4P model.

Started in 2010, the key focus of P4P project is to improve provider performance and enhance the quality of services of the institution as a whole. Dr. Haque noted that P4P was successful in most of the facilities while in a few facilities the program was not successful. He emphasized to identify the gaps in service delivery within the system, which should be met, but program should not be stopped. Dr. Haque suggested adopting DSF lessons for P4P in increasing community awareness and simultaneously reaching mothers through facilities located at the community level. Like DSF, P4P should be reformed for its future continuation.

There are two concerns in the $\mathrm{P} 4 \mathrm{P}$, which require attention: to incorporate a mechanism for measuring individual performance, and to consider the overall performance of a facility in case of non-performance of certain providers so that the facility might not be deprived from receiving incentives. Dr. Haque emphasized on the effective leadership of managers, where they will be responsible for the performance of respective facility. If necessary, training should be in place for managers to enhance their leadership and managerial skills.

Along with performance reward, Dr. Haque felt the necessity to introduce disincentive for negligence and absenteeism of service providers or for poaching patients to private clinics. He was not supportive of universal roll-out of performance-based incentives. Instead, incentive should be given to providers if they work beyond duty hour. Dr. Haque emphasized to identify a mechanism of where or in what capacity performance-based financing is needed. Whatever the mechanism, availability of providers is the key challenge.

Dr. Haque concluded his speech with optimism that recommendations of the workshop would shape policy development for performance-based financing in the health sector of Bangladesh. 


\section{Speech of the chief guest}

Mr. Md. Humayun Kabir, Secretary, MOHFW began his speech by emphasizing the importance to implement innovative approaches for improving the service delivery and thereby increasing utilization of services from health facilities towards reducing maternal and neonatal deaths, but he underlined the need to carry out independent evaluation of such programs prior to mainstreaming their good practices. The Secretary stated that such programs should not continue for an indefinite time. It is necessary to decide which program to continue and in what capacity.

The Secretary highlighted some achievements of both DSF and P4P programs. Incentive linked to the performance of an institution practiced in $\mathrm{P} 4 \mathrm{P}$ was more appealing to the Secretary than the case-based incentive to direct providers as practiced in DSF. He emphasized modification of DSF program, in case of further expansion.

Shortage of human resources is a long-standing challenge to the health system in Bangladesh. If obstetrician-anesthesiologist pair is not ensured at upazila hospital, DSF and P4P programs will not be able to generate significant impact. The Secretary strongly felt the need to rationalize the posting of specialized doctors (obstetrician and anesthesiologist) towards achieving MDG 5.

The Secretary expressed his interest in continuing incentive-based programs like DSF or P4P as he reasoned that these programs are still in trial phase and valued for their contribution towards increasing institutional delivery. He particularly emphasized to continue with DSF and suggested for an evaluation of P4P program. But, he questioned the sustainability of such donor-supported performance-based financing programs. The government is not capable of supporting those projects from its own resources, for which the Secretary felt the need to seek alternative source of financing.

User fee has the potential to increase resources in the health sector. The Secretary, however, expressed his concerns with the centralized financial control. The Ministry of Finance manages the money that is collected from the clients in exchange of services. It is necessary to find a mechanism, which would be acceptable to both the Ministry of Health and Family Welfare and the Ministry of Finance. It is only seven percent of national budget allocated to the health sector, which is grossly inadequate for its proper functioning. The Secretary suggested that public-sector allocations to health need to be increased with due recognition to performance.

In the process of preparing a health financing strategy, it is necessary to consult with concerned government agencies to reach a consensus for using local resources. The Secretary recommended incorporating "community participation" as a strategy to generate resources in health financing strategy. He cited the community contribution in establishing Community Clinics, particularly in donating land and mobilizing fund.

The Secretary thanked Population Council for organizing such a technical and timely workshop aimed at shaping the future performance-based financing programs to improve maternal, newborn and child health in Bangladesh. 


\section{WAY FORWARD}

To improve access to facility-based obstetric and newborn care, the Government of Bangladesh is implementing two innovative performance-based financing programs, namely DSF and P4P. Both the programs contributed to the increase in institutional deliveries. Despite financial incentive in the public sector a new phenomenon in Bangladesh, the necessity for introducing performance-based incentive has been justified through the positive results of both DSF and P4P programs.

Nevertheless, the most pressing challenge for future of performance-based incentive will be securing sustainable financing. Both DSF and P4P are positive developments, for which the government needs to prepare itself by taking adequate measures in resource mobilization. In addition, it is necessary to draft ways to incorporate changes into the DSF scheme or to modify P4P for further expansion. The following discussion highlights the modifications suggested at the workshop for the DSF and P4P programs.

\section{Modification of DSF scheme}

DSF is the first attempt in the health sector of Bangladesh that provides financial support to poor women for receiving safe delivery services from facilities in rural areas. Supply side is also financially benefited through this program. Service providers receive case-based incentive, which is provided monthly, calculating the total number of services provided to poor pregnant women. The key recommendations from the workshop to strengthen the DSF program are:

\section{Incentive beneficiary}

- Incentives should be given to motivate service providers at the institution level, instead at the individual level as currently practiced in DSF. In other word, incentive should be provided to both direct and indirect providers as well as management, administrative and support staff of a facility.

- At the supply side, there is a missed opportunity for the DSF scheme that providers working in the obstetric unit receive the incentive while service providers working at other units are not included as beneficiary. Whole institution approach can be implemented, i.e., all units of a facility should be brought under the umbrella of DSF. P4P's team approach can be adopted in covering the whole institution.

- At the demand side, DSF aims to provide financial assistance to only pregnant women for receiving obstetric care services. DSF should also target neonate and under-five children's services in addition to the services for pregnant women.

\section{Performance measurement criteria}

- Only quantity (number of services provided to the voucher clients) is considered to measure the performance of providers in the DSF scheme. Inclusion of quality as a measurement 
indicator is required urgently. Provider performance should be linked with both quantity and quality of services.

- Performance should be measured quarterly replacing the current monthly measurement system.

\section{Performance measurement system}

- DSF needs to introduce a systematic quality assurance mechanism to measure the performance of facility on a regular basis. Additionally, such system will provide an opportunity to impart supportive feedback to the providers for improving services.

\section{Quality assurance system}

Quality assurance system implemented under P4P project is useful in identifying gaps in service delivery and providing mentoring on a regular basis. DSF scheme can adopt the P4P experiences of introducing both external and internal quality assurance system.

- For external quality assurance system, DSF needs to form quality assurance groups (QAGs) within each district, where faculty from Medical College or consultants from District Hospital may visit Upazila Health Complex with comprehensive emergency obstetric care and consultants from District Hospital for Upazila Health Complex with basic emergency obstetric care.

- As part of internal quality assurance system, within the facility, unit-based quality assurance teams (QATs) can be formed to monitor and review performance routinely and ensure coordination between team members.

\section{Payment mechanism}

Under the DSF scheme, only providers receive incentives while managers and administrative staff are not covered as beneficiary. Other problems with payment mechanism which need immediate attention include:

- Increase amount of benefits for both providers and service recipients.

- Introduce referral fees for skilled birth attendant to promote institutional delivery.

- Provide financial benefits to skilled birth attendant for performing delivery at home.

- Reduce the gap in incentive amount between surgeon and anesthesiologist. 


\section{Modification of P4P model}

$\mathrm{P} 4 \mathrm{P}$ project is a human resource innovation project - paying an incentive to the institution for achieving pre-determined targets. Incentives are provided to motivate service providers at the institution level, which in turn improves provider availability. Providing incentives to management, service, administrative and support staff based on their level of efforts contributed to ensure teamwork as a way to improve outcomes, which is a unique characteristic of the P4P approach. P4P approach contributed in improving the quality of care while increasing the quantity of services. P4P is an effective approach as provider performance has been linked with both quantity and quality of services. However, the workshop resulted in several recommendations for future expansion of the P4P approach.

\section{Implementation facility}

P4P was implemented at selected Upazila Health Complexes and District Hospitals, leaving an opportunity to incorporate other facilities under the district health system for future expansion. Regarding implementation facility, two specific suggestions were made.

- Mother and Child Welfare Centers and union Health and Family Welfare Centers should be included.

- A whole district approach (all upazilas with both uapzila- and union-level facilities) should be followed in phased manner.

\section{Incentive beneficiary}

- Not all units perform equally. The eligibility of a facility to receive performance bonuses largely depends on the obstetric unit. Despite weak functioning and unsatisfactory performance of other units, performance of obstetric unit helps the facility to get incentives. Non-performers should not be automatically provided incentive.

- At present, both government and non-government fieldworkers are included as beneficiary for referral services but their supervisors (e.g., assistant health inspector, health inspector and family planning inspector) are not entitled as beneficiary. It is necessary to incorporate them as beneficiary at the supply side.

- Providing financial assistance to poor clients is strongly recommended. There should be an integrated strategy in P4P project - both demand and supply side incentives should be provided.

\section{Performance measurement criteria}

- Family planning unit of a facility does not have separate targets and responsibilities. They automatically become eligible to receive the incentive once the health unit achieves the performance targets. It is urgently required to introduce separate targets for the family planning unit. 
- Health unit of a facility manages several deadly complications related to pregnancy and delivery which are not included as quantitative targets. It is necessary to include new indicators, e.g., number of death, eclampsia management, removal of retained placenta, management of ante-partum and post-partum hemorrhage, dilation and curettage, and post abortion care.

- To sustain performance momentum, it is critical to establish a reward system for outstanding performer along with the existing incentive mechanism.

\section{Performance measurement authority}

- P4P project management committee is composed of six members, with four members from the respective facility, but not having any consultants as its members. These committees should include Obstetrics, Pediatrics, and Anesthesiology Consultants (if any) of the respective facility as its members.

- Empowering facility managers is critical so that they can reward the outstanding performer, undertake local recruitment, and adopt corrective measure for non-performers.

\section{Quality assurance system}

The uniqueness of P4P project lies in its quality assurance system. Yet, there is scope for improving the system for future expansion.

- Quality assurance checklist should be abridged and automated.

- Expanding the quality assurance system at all upazilas will be a big challenge due to limited availability of experts from tertiary level hospitals. In the interim period, a QAG may be opted for certain number of facilities. Alternatively, there might be supervision from medical college to district hospital and from district hospital to upazila hospital.

- Existing rate of honorarium for the QAG members for visiting health facilities should be increased by considering the distance and the opportunity cost.

\section{Programmatic challenges}

Both DSF and P4P have shown promises in rapid reduction of maternal deaths, but they are laden with a risk of sustainability. These programs are still in trial phase and valued for their contribution towards increasing institutional delivery. Nevertheless, human resources, sustainable funding and delegation of authority remain as the key challenges.

\section{Human resources}

Incentive may be counterproductive if required manpower, training and monitoring are not in place. In such cases, money will not lead to willingness, rather morality may be waned. The most critical challenge to sustain the performance-based financing programs for maternal, newborn and child 
health services in Bangladesh is the shortage of human resources, especially obstetrician and anesthesiologist pair. Highest importance should be given to ensure obstetrician and anesthesiologist pair at the upazila hospital; otherwise, DSF and P4P programs will not be able to generate significant impact. Rationalizing the posting of specialized doctors towards achieving MDG 5 is urgently required, i.e., to ensure the availability of anesthesiologist alongside an obstetrician, for which several strategies are important:

- Continue essential obstetric care training.

- Create more number of anesthesiologists.

- Ensure no transfer or deputation during project period, without having appropriate substitute.

There is no system of disincentive for poor performers in both DSF and P4P programs. For any incentive-based financing program, there should be two sides, one is reward and the other is punishment. It will be difficult to keep the better performers' commitment if there is no system of disincentive for negligence, non-cooperation and absenteeism of service providers or for diverting patients to private clinics. While better performers should be rewarded, poor performers need to be excluded from the incentive package.

\section{Alternative health care financing}

Sustainability of performance-based financing programs, which are now supported by development partners, is a critical challenge. Performance-based financing is not an alternative approach to health financing, as it does not generate any revenue rather it requires a large investment. The government is not capable of supporting such projects from its own resources, for which "user fee" can be introduced as an alternative source of financing. Although user fee has the potential to increase resources in the health sector, it has a great risk - poverty remains as the key obstacle to mainstream such initiative. In such cost-sharing mechanism, poor families in rural areas should not only be exempted from user fees, but also be given financial assistance in seeking health care.

Resource mobilization through community involvement can also be an effective tool for sustaining a program. Reactivating health management committees at different tiers of health-system service delivery may prompt a sense of ownership among community people, if programs are jointly managed by the government and local people. If DSF and P4P could reactivate health management committees, the community might own those programs in future.

At present, about seven percent of national budget is allocated to the health sector, which is grossly inadequate for its proper functioning. Public-sector allocations to health need to be increased with due recognition to performance. 


\section{Delegation of authority}

Central management requires a long process to make any recruitment and procurement for the health facilities in rural areas. It is necessary to grant greater managerial and financial autonomy to District Hospitals and Upazila Health Complexes. As an interim measure, Civil Surgeon should be allowed to rearrange the workforce across Upazila Health Complexes, so that every Upazila Health Complex will have minimum required staff-mix. Concurrently, Upazila Health and Family Planning Officer should be empowered to undertake local recruitment and to discipline staff. Specifically, they should have full autonomy in the recruitment of several groups of workers, particularly support staff.

Centralized financial control undermines the effectiveness of cost recovery. The Ministry of Finance manages the money that is collected from the clients in exchange of services. Until recently, the revenues generated from user fees are returned to the state treasury, with barely any impact upon the quality of health care of facilities. Local hospitals should be allowed to keep and spend the user fees that they collect rather than return them to the state treasury. Empowering local managers is critical to retain the user fees for sustainable improvement of quality of services.

\section{What next}

Performance-based incentive mechanism is an innovative strategy to tackle issues related to service use and provider performance. It is a transitional mechanism to expedite health results. In Bangladesh, the need for continuing performance-based financing programs to meet MDGs and other health indicators is beyond argument. Specifically, such encouragement is required until certain level of institutional deliveries and improvement in maternal and child health are reached. It is urgently required to decide in what capacity performance-based financing program should continue.

Whatever the approach may be, the necessity of "quality of care" approach to improve maternal, newborn and child health services tested under the P4P scheme cannot be denied for the improvement of the monitoring of health service delivery in Bangladesh. Particularly, P4P's quality assurance approach may have implications to enrich the DSF scheme for performing better. It will be more useful and feasible if policymakers and program managers adopt results from both DSF and $\mathrm{P} 4 \mathrm{P}$ and advance with a revised model, which will require less money and be more effective in pursuance of achieving MDGs of reducing maternal and neonatal mortality. 


\section{ANNEXURE}

\section{Program of the workshop}

\section{National Workshop on}

Innovative Financing through Pay-for-Performance for Providers to Improve Quality of Care in Bangladesh: Transforming Research into Action

12-13 December 2011, Lake Shore Hotel, Dhaka

12 December 2011 (Monday)

Chief Guest: Mr. Md. Humayun Kabir, Secretary, MOHFW

Chair: Dr. Md. Shamsul Haque, Director, Primary Health Care, DGHS

\begin{tabular}{|c|c|}
\hline 9:30 am - 10:00 am & Registration with Morning Refreshments \\
\hline 10:00 am - 10:10 am & $\begin{array}{l}\text { Welcome Speech and Purpose of the Workshop } \\
\text { Dr. Md. Shamsul Haque, Director, Primary Health Care, DGHS }\end{array}$ \\
\hline $10: 10 \mathrm{am}-10: 30 \mathrm{am}$ & $\begin{array}{l}\text { Performance Based Financing- Theory and Examples on International Experiences } \\
\text { Dr. Ubaidur Rob, Country Director, Population Council }\end{array}$ \\
\hline $10: 30 \mathrm{am}-10: 50 \mathrm{am}$ & $\begin{array}{l}\text { Health Care Financing Strategy Process in Bangladesh } \\
\text { Mr. Prasanta Bhushan Barua, Joint Chief, HEU, MOHFW }\end{array}$ \\
\hline 10:50 am - 11:10 am & $\begin{array}{l}\text { DSF Scheme: Design, Experiences, Challenges and Way Forward } \\
\text { Dr. Azizul Alim, DGHS, MOHFW }\end{array}$ \\
\hline $11: 10 a m-11: 50 a m$ & $\begin{array}{l}\text { P4P Model: Context, Design, Experiences, Challenges and Way Forward } \\
\text { Dr. Riad Mahmud, UNICEF and Laila Rahman, Population Council }\end{array}$ \\
\hline 11:50 am - 12:20 pm & $\begin{array}{l}\text { Testimonies of the Implementers: } \\
\text { P4P Committees, Quality Assurance Groups, } \\
\text { Quality Assurance Teams and Institutional Beneficiaries }\end{array}$ \\
\hline $12: 20 \mathrm{pm}-1: 00 \mathrm{pm}$ & Open Discussion \\
\hline 1:00 pm - 1:10 pm & $\begin{array}{l}\text { Speech by the Special Guest } \\
\text { Dr. Mohammed Sharif, Line Director, MCH, DGFP }\end{array}$ \\
\hline 1:10pm-1:20pm & $\begin{array}{l}\text { Speech by the Special Guest } \\
\text { Mr. Michel Saint-Lot, Deputy Representative, UNICEF }\end{array}$ \\
\hline $1: 20 p m-1: 30 p m$ & $\begin{array}{l}\text { Remarks by the Chair } \\
\text { Dr. Md. Shamsul Haque, Director, Primary Health Care, DGHS }\end{array}$ \\
\hline $1: 30 \mathrm{pm}$ & Lunch Follows \\
\hline
\end{tabular}




\title{
Program (Continued)
}

\section{National Workshop on}

Innovative Financing through Pay-for-Performance for Providers to Improve Quality of Care in Bangladesh: Transforming Research into Action

\author{
12-13 December 2011, Lake Shore Hotel, Dhaka
}

13 December 2011 (Tuesday)

Chief Guest: Mr. Md. Humayun Kabir, Secretary, MOHFW

Chair: Dr. Md. Shamsul Haque, Director, Primary Health Care, DGHS

\begin{tabular}{|c|c|}
\hline 9:00 am - 9:30 am & Registration with Morning Refreshments \\
\hline 9:30 am - 10:00 am & $\begin{array}{l}\text { Setting the Stage for Second Day's Activities of the Workshop } \\
\text { - Video Clip: Ray of Hope with Pay-for-Performance Innovation } \\
\text { in Bangladesh } \\
\text { - Purpose and recapitulation of the first day } \\
\text { - Group formation and group work instructions }\end{array}$ \\
\hline 10:00 am - 11:30 am & $\begin{array}{l}\text { Group Work } \\
\text { - Group A: Incorporation of the quality of care framework and modification of the } \\
\text { financing mechanism in the DSF scheme } \\
\text { - Group B: Modification of the institution-based P4P model }\end{array}$ \\
\hline $11: 30 \mathrm{am}-12: 45 \mathrm{pm}$ & Group Work Presentation and Open Discussion \\
\hline $\begin{array}{l}11: 30 \mathrm{am}-11: 45 \mathrm{am} \\
11: 45 \mathrm{am}-12: 00 \mathrm{pm} \\
12: 00 \mathrm{pm}-12: 45 \mathrm{pm}\end{array}$ & $\begin{array}{l}\text { Group A :Presentation } \\
\text { Group B: Presentation } \\
\text { Open Discussion }\end{array}$ \\
\hline $12: 45 p m-12: 55 p m$ & $\begin{array}{l}\text { Speech by the Special Guest } \\
\text { Mr. Prasanta Bhushan Barua, Joint Chief, HEU, MOHFW }\end{array}$ \\
\hline $12: 55 p m-1: 15 p m$ & $\begin{array}{l}\text { Speech by the Chief Guest } \\
\text { Mr. Md. Humayun Kabir, Secretary, MOHFW }\end{array}$ \\
\hline 1:15 pm-1:25 pm & $\begin{array}{l}\text { Closing Remarks by the Chair } \\
\text { Dr. Md. Shamsul Haque, Director, Primary Health Care, DGHS }\end{array}$ \\
\hline $1: 25 \mathrm{pm}-1: 30 \mathrm{pm}$ & $\begin{array}{l}\text { Vote of Thanks } \\
\text { Dr. Md Sayedur Rahman, Program Manager, RH, DGHS }\end{array}$ \\
\hline $1: 30 \mathrm{pm}$ & Lunch Follows \\
\hline
\end{tabular}




\section{List of participants}

(Not according to seniority)

Mr. Md. Humayun Kabir

Secretary, Ministry of Health and Family Welfare, Government of Bangladesh

Mr. Prashanta Bhushan Barua,

Joint Chief, Health Economics Unit, Ministry of Health and Family Welfare

Dr. Md. Shamsul Haque

Director, Primary Health Care, Directorate General of Health Services

Dr. Deen Mohammad

Deputy Director, Primary Health Care, Directorate General of Health Services

Dr. Md. Sayedur Rahman

Program Manager, Reproductive Health, Directorate General of Health Services

Dr. Ishita H. Chowdhury

Deputy Program Manager, Reproductive Health, Directorate General of Health Services

Dr. Md.Azizul Alim

Deputy Program Manager, Essential Obstetric Care, Directorate General of Health Services

Dr. Akhtar Jahan

Assistant Director, Maternal and Newborn Health, Directorate General of Health Services

Dr. Shelina Afroza

Director General, National Institution for Population Research and Training (NIPORT)

Dr. Md. Shahidul Huq

Civil Surgeon, Gaibandha

Dr. A.B.M. Abu Hanif

Resident Medical Officer, Gaibandha District Hospital

Dr. Md. Yousuf Harun-or-Rashid

Consultant (Pediatrics), Gaibandha District Hospital

Dr. Rokshana Rahat

Assistant Surgeon, Gaibandha District Hospital

Dr. Md. Aminul Islam

Upazila Health and Family planning Officer, Shaghata Upazila Health Complex, Gaibandha

Dr. Robin Faysal

Resident Medical Officer, Shaghata Upazila Health Complex, Gaibandha

Dr. Mosnet Ara Begum

Upazila Health and Family Planning Officer, Sundarganj Upazila Health Complex, Gaibandha

Dr. Moloy Kumar Roy

Resident Medical Officer, Sundarganj Upazila Health Complex, Gaibandha

Dr. Amal Chandra Saha

Upazila Health and Family Planning Officer, Fulchari Upazila Health Complex, Gaibandha 
Dr. Debashish Mondol Angkur

Resident Medical Officer (In-charge), Fulchari Upazila Health Complex, Gaibandha

Dr. Reazul Islam

Civil Surgeon, Kurigram

Dr. Lokman Hakim

Deputy Civil Surgeon, Kurigram

Dr. Md. Nazrul Islam

Resident Medical Officer, Kurigram District Hospital

Dr. Md. Mustafizur Rahman

Junior Consultant (Gynecology/Obstetrics), Kurigram District Hospital

Dr. Mokhtar Ali

Junior Consultant (Pediatrics), Kurigram District Hospital

Dr. Md. Shamsuddoha

Deputy Director-Family Planning, Kurigram

Dr. Md. Motasim Billah Azad

Upazila Health and Family Planning Officer, Chilmari Upazila Health Complex, Kurigram

Dr. Md. Saifur Rahman

Upazila Health and Family Planning Officer, Bhurungamari Upazila Health Complex, Kurigram

Dr. Md. Alim Sarker

Resident Medical Officer, Bhurungamari Upazila Health Complex, Kurigram

Dr. G.R.M. Moksedur Rahman

Upazila Health and Family Planning Officer, Nageshwari Upazila Health Complex, Kurigram

Dr. Md. Amzad Hossain

Resident Medical Officer, Nageshwari Upazila Health Complex, Kurigram

Dr. Narayan Chandra Dey

Civil Surgeon, Jamalpur

Dr. Jatindra Chandra Mandal

Resident Medical Officer, Jamalpur District Hospital

Dr. Fakhria Alam

Consultant (Gynecology/Obstetrics), Jamalpur District Hospital

Dr. Md. Tazul Islam

Junior Consultant (Pediatrics), Jamalpur District Hospital

Dr. Md. Feroze Khan

Upazila Health and Family Planning Officer, Islampur Upazila Health Complex, Jamalpur

Dr. Mohammad Zahir Uddin

Resident Medical Officer (In-charge), Islampur Upazila Health Complex, Jamalpur

Dr. Md. Gias Uddin

Upazila Health and Family Planning Officer, Melandah Upazila Health Complex, Jamalpur

Dr. Mehedi Iqbal

Resident Medical Officer, Melandah Upazila Health Complex, Jamalpur 
Dr. Md. Shamsul Haque

Upazila Health and Family Planning Officer, Bakshiganj Upazila Health Complex, Jamalpur

Dr. Md. Hibrul Bari

Resident Medical Officer, Bakshiganj Upazila Health Complex, Jamalpur

Dr. Nurul Islam

Representative of Civil Surgeon, Barisal

Dr. Md. Emdad Haque

Junior Consultant (Gynecology/Obstetrics), Chowgacha Upazila Health Complex, Jessore

Dr. Md. Abdur Rahim,

Upazila Health and Family Planning Officer, Kalapara Upazila Health Complex, Patuakhali

Dr. Md. Emran

Upazila Health and Family Planning Officer, Bhanga Upazila Health Complex, Faridpur

Dr. S.M. Abdul Jalil

Upazila Health and Family Planning Officer, Gobindoganj Upazila Health Complex, Gaibandha

Dr. Md. Reajul Islam

Upazila Health and Family Planning Officer, Baliadangi Upazila Health Complex, Thakurgaon

Dr. Md. Nazrul Islam

Upazila Health and Family Planning Officer, Shyamnagar Upazila Health Complex, Shatkhira

Dr. Matiur Rahman

Upazila Health and Family Planning Officer, Shibchar Upazila Health Complex, Madaripur

Dr. Md. Golam Faruk

Upazila Health and Family Planning Officer, Naria Upazila Health Complex, Shariatpur

Dr. Muhammed Imran Uddin

Resident Medical Officer, Matlab (North) Upazila Health Complex, Chandpur

Dr. Md. Jafar Islam

Medical Officer, Daulatpur Upazila Health Complex, Khulna

Dr. Sanjay Kumar Mondal

Medical Officer, Paikgacha Upazila Health Complex, Khulna

Mr. Omar Sharif Shibly

DSF Coordinator, WHO, Ukhia, Cox's Bazar

Mr. Md. Ashrafuzzaman

DSF Coordinator, WHO, Shahjadpur, Sirajgonj

Mr. Kabir Hossain

DSF Coordinator, WHO, Shibchar, Madaripur

Mr. Rabindranath Adhikary

DSF Coordinator, WHO, Harirampur

Dr. Ashima Bhowmik

Assistant Professor (Gynecology/Obstetrics), Rangpur Medical College

Dr. Anisa Begum

Assistant Professor (Gynecology/Obstetrics), Rangpur Medical College 
Dr. Aziza Begum

Assistant Professor (Gynecology/Obstetrics), Rangpur Medical College

Dr. Md. Ataur Rahman

Assistant Professor (Anesthesiology), Rangpur Medical College

Dr. Sayedur Rahman

Assistant Professor (Anesthesiology), Rangpur Medical College

Dr. Md. Jahidur Rahman

Assistant Professor (Pediatrics), Rangpur Medical College

Dr. Laila Husna Banu

Professor (Gynecology/Obstetrics), Prime Medical College \&

OGSB Representative, Rangpur

Dr. S.M. Shahida

Assistant Professor (Gynecology/Obstetrics), Mymensingh Medical College

Dr. Md. Shafiqul Islam

Assistant Professor (Pediatrics), Mymensingh Medical College

Dr. A.K.M. Sadiqul Azam

Assistant Professor (Gynecology/Obstetrics), Mymensingh Medical College \&

OGSB Representative, Mymensingh

Mr. Dhiraj Kumar Nath

Consultant, Asian Development Bank

Dr. Jan Borg

Health Advisor, AusAid

Mr. Sharukh Safi

Senior Program Officer, AusAid

Dr. Momena Khatun

Health Advisor, Canadian International Development Agency (CIDA)

Ms. Khadijat Mojidi

Director, Office of Population, Health and Nutrition, USAID

Ms. Shanon Young

Health Officer, USAID

Mr. Yogesh Rajkotia

USAID

Mr. Yann Derriennic

Health System 20/20, USAID

Dr. Shehlina Ahmed

Health and Population Advisor, DFID

Ms. Yukie Yoshimura

Chief Advisor, Safe Motherhood Promotion Project, JICA

Mr. Habibur Rahman

Senior Program Manager, KFW 
Ms. Tahmina Begum

Consultant, World Bank

Mr. Naveed Ahmed

Consultant, World Bank

Mr. Pierre-Yves Lambert

Program Manager, Health, European Union

Mr. Michel Saint-Lot

Deputy Representative, UNICEF

Dr. Riad Mahmud

Health Officer, UNICEF

Mr. Yuki Suehiro

Deputy Representative, UNFPA

Mr. Touhid Alam

SPO-Strategic Direction, UNFPA

Mr. Aminul Arefeen

Technical Officer, M\&E and Research, UNFPA

Mr. Joshua Bryant

Consultant, UNFPA

Mr. Abdur Razzaque

Senior M\&E Assistant, WHO

Mr. Md. Ashraful Alam

DSFC, WHO

Ms. Laura Reichenbach

Director, Centre for RH, ICDDR,B

Dr. Shamim Jahan

Technical Director, FHI 360

Dr. S.K. Asiruddin

Team Leader, Traction/URC

Dr. Lars Chr. Kyburg

Team Leader, GFA

Dr. Palak Mutsuddy

Health Economist, GFA

Dr. Selina Amin

Country Project Manager, Plan Bangladesh

Dr. Razzaqul Alam

Health Specialist, Plan Bangladesh

Ms. Shamima Sultana

Program Officer, Engender Health

Dr. M.H. Choudhury

General Manger (Services), Marie Stopes Bangladesh 


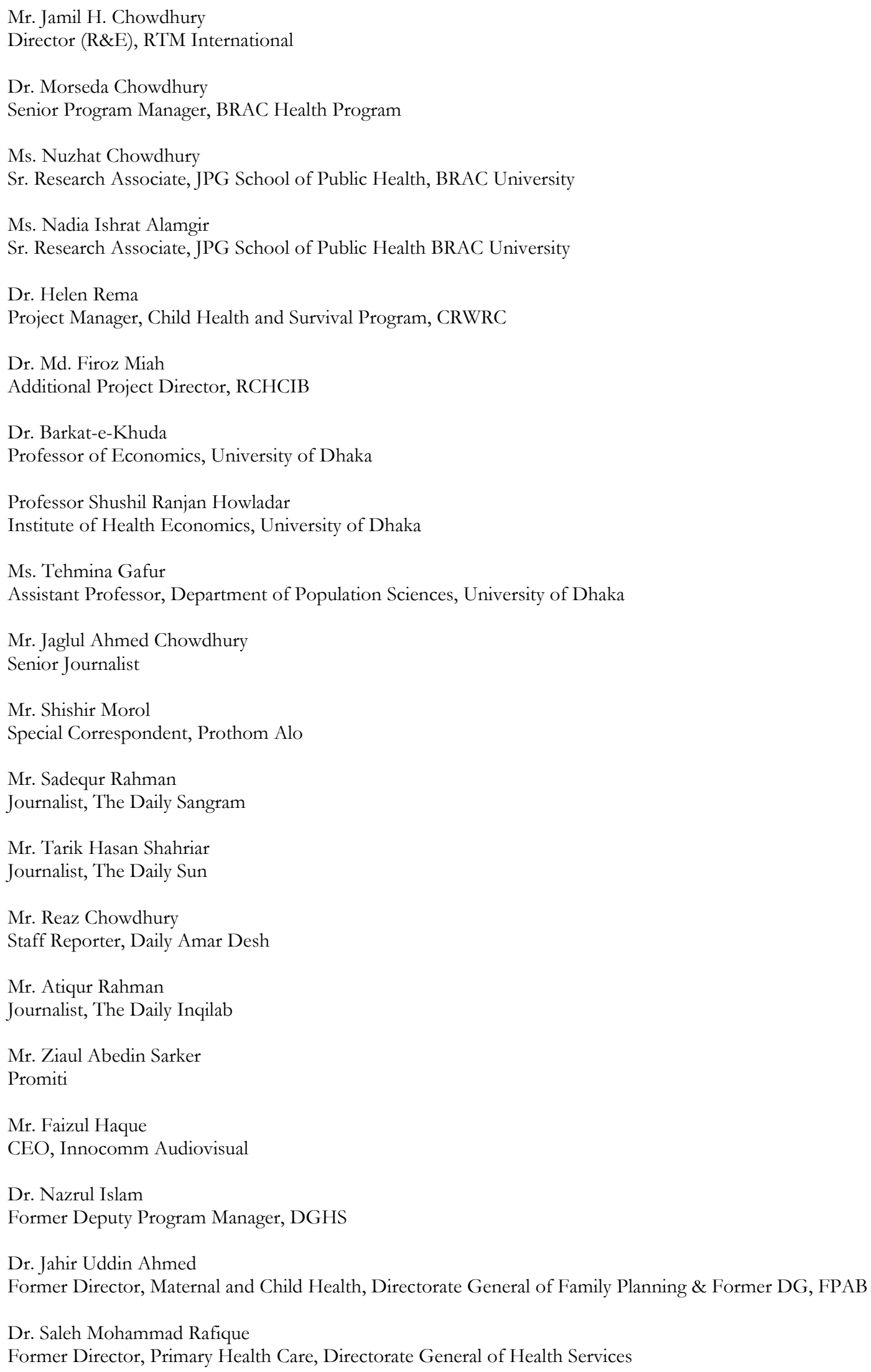


Dr. A.B.M. Jahangir Alam

Former Director, Primary Health Care, Directorate General of Health Services

Mr. Liton Acharja

Research Officer, Population Council

Mr. Arifur Rahman

Research Officer, Population Council

Mr. Anup Kumar Dey

Research Officer, Population Council

Mr. Abdur Rab Sarder

Research Officer, Population Council

Ms. Nargis Sultana

Research Officer, Population Council

Mr. Md. Julkernayeen

Senior Research Officer, Population Council

Mr. Ataur Rahman

Senior Research Officer, Population Council

Mr. Md Hafizur Rahman

Assistant Program Officer, Population Council

Mr. Md. Noorunnabi Talukder

Program Officer, Population Council

Mr. Md. Moshiur Rahman

Program Officer, Population Council

Dr. Ismat Ara Hena

Program Officer, Population Council

Ms. Laila Rahman

Senior Program Officer, Population Council

Mr. A.K.M. Zafar Ullah Khan

Advisor, Population Council \& Former Secretary, MOHFW

Dr. Ubaidur Rob

Country Director, Population Council 


\section{Press coverage}

\section{dailiy sutili}

DHAKA | Tuesday | 13 December 2011 | 29 Agrahayan 1418 BS | 17 Muharram 1433 HIJRI

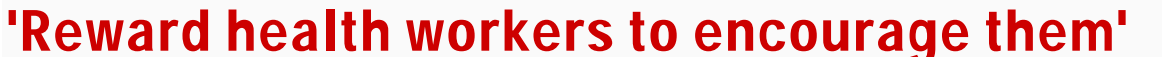 \\ Staff Correspondent}

Introduction of reward for performance would encourage health workers to carry out their responsibilities properly, speakers said at a workshop on Monday.

Besides, the government should ensure punishment of those employees of health and family planning departments who do not perform their duties properly and are absent from workplaces, they said.

The Directorate General of Health Services (DGHS), Population Council and Unicef jointly orgainsed the workshop titled 'Innovating financing through pay-for-performance for providers to improve quality of care in Bangladesh: Transforming research into action' at Lakeshore Hotel in the city.

While addressing the programme, Population Council's Country Director Dr Obaidur Rob said the country would face more challenges of providing health care services to people in near future if the present population growth rate is not considered. Every year two million people are being added to the population, he said, adding that more health care resources will be needed for this additional people. He urged the health workers to motivate people living in remote areas to keep their family size small.

Prasanta Bhushan Barua, joint chief of Health Economics unit under the Health and Family Welfare Ministry, said poor people are still not getting adequate health care services across the country due to poverty. Free health care services needs to be introduced across the country for the poor, he said.

Dr Shamsul Haque, director of Primary Health Care of DGHS, said introduction of performance based reward for health workers in Gaibandha, Jamalpur and Kurigram is producing positive results. He said the reward programme needs to be extended to Char, costal and hilly areas to make health care services available to the poor people.

Former adviser to caretaker government DK Nath, Barkat-e-Khuda, professor of Economics Department at Dhaka University, and Michel Saint-Lot, deputy country representative of Unicef, addressed the programme. 


\title{
• আমার $\square$ দেশ \\ স্বাধীনতার কথা বলে
}

ঢাকা, মঙ্গলবার ১৩ ডিসেম্বর ২০১১, ২৯ অগ্রহায়ণ ১৪১৮, ১৭ মহররম ১৪৩২

\section{কর্মশালায় স্বাস্থ্য বিশেষজ্ঞরা : পে ফর পারফরম্যান্স স্বাস্থ্যসেবায় নতুন অধ্যায় সৃষ্টি করবে}

\author{
স্টাফ রিপোর্টার
}

পারফরম্যান্স- ভিত্তিক প্রণোদনা প্রদান দেশের স্বাস্থ্যসেবায় নতুন অধ্যায় সৃষ্টি করবে। টার্গেট অনুযায়ী সেবা প্রদান করলে হাসপাতাল থেকে সেবা গ্রহণের হার উল্লেখযোগ্যভাবে বৃদ্ধি পাবে, সেইসঙ্গে সেবার গুণগত মানও বৃদ্ধি পাবে। এতে সেবা প্রদানকারী এবং সেবা গ্রহণকারী উভয়েই উপকৃত হবে।

গতকাল রাজধানীর একটি হোটেলে পে ফর পারফরম্যান্স বিষয়ক দুদিনব্যাপী এক কর্মশালার উদ্বোধনী সেশনে স্বাস্থ্য বিশেষজ্ঞরা এসব কথা বলেন। কর্মশালায় বিশেষজ্ঞরা জানান, রুয়াল্ডা, বিলিজ, কেনিয়া, হাইতি, কম্বোডিয়া, আফগানিস্তানসহ পৃথিবীর অনেক দেশেই পে ফর পারফরম্যান্স প্রচলিত আছে। দেশে এই পে ফর পারফরম্যান্স কার্যক্রম খুবই নতুন। পপুলেশন কাউন্সিল পাইলট পর্যায়ে স্বাস্থ্য অধিদফতরকে গাইবান্ধা, কুড়িগ্রাম ও জামালপুর জেলায় পরীক্ষামূলক পে ফর পারফরম্যান্স কার্যক্রম বাস্তবায়নে কারিগরি সহায়তা প্রদান করছে।

স্বাস্থ্য অধিদফতর এবং পপুলেশন কাউন্সিলের যৌথ উদ্যোগে আয়োজিত এ কর্মশালায় সভাপতিত্ব করেন প্রাইমারি হেলথ কেয়ারের পরিচালক ডা. নো. শামসুল হক। পে ফর পারফরম্যান্স বিষয়ক আন্তর্জাতিক অভিজ্ঞতাগুলো তুলে ধরেন পপুলেশন কাউন্সিলের কান্ট্রি ডিরেক্টর ড. ওবায়দুর রব। কাউন্সিলের সিনিয়র প্রোগ্রাম অফিসার লায়লা রহমান বাংলাদেশে পে ফর পারফরম্যান্সের পাইলট প্রজেক্টের বাস্তবায়ন, ফলাফল এবং অর্জিত অভিজ্ঞতার ওপর একটি তথ্যপূর্ণ প্রতিবেদন উপস্থাপন করেন। উদ্বোধনী অনুষ্ঠানে অন্যদের মধ্যে বক্তব্য রাখেন পরিবার পরিকল্পনা অধিদফতরের এমসিএইচের লাইন ডিরেক্টর ডা. মোহাম্মদ শরীফ এবং ইউনিসেফের ডেপুটি রিপ্রেজেন্টেটিভ মিশেল সেইন্ট- লট, তত্ত্বাবধায়ক সরকারের সাবেক উপদেষ্টা ধীরাজ কুমার নাথ, স্বাস্থ্য মন্ত্রণালয়ের হেলথ ইকোনমিক্স ইউনিটের যুগ্ম প্রধান প্রশান্ত ভূষণ বড়য়া, স্বাস্থ্য অধিদফতরের ডা. আজিজুল আলীম, ইউনিসেফের ড. রিয়াদ মাহমুদ এবং পপুলেশন কাউন্সিলের অ্যাডভাইজার একেএম জাফর উল্লাহ খান।

অনুষ্ঠানে জানানো হয় পে ফর পারফরম্যান্স হলো লেবা প্রদানকারী প্রতিষ্ঠান এবং লেবাপ্রদানকারীকে আর্থিক সুবিধা প্রদানের মাধ্যমে কর্মদক্ষতা এবং কর্মসম্পাদনের হার বৃদ্ধিতে উতসাহ দেয়া। পে ফর পারফরম্যান্স মডেলে প্রতিষ্ঠানকে প্রণোদনা প্রদানের মাধ্যমে তাদের পারফরম্যান্স বূদ্ধির প্রয়াস থাকায় সেবা গ্রহীতাও মানসম্পন্ন সেবা পেয়ে থাকেন। বিশেষ করে দুর্গম ও পশচাতপদ এলাকা, লো- পারফর্মিং এলাকা, চর কিংবা হাওর এলাকার জন্য এ মডেল খুবই গুরুত্বপূর্ণ। 


\section{व्रथव्व आल्या}

ঢাকা, ১৩ ডিসেম্বর ২০১১, ২৯ অগ্রহায়ণ ১৪১৮, ১৭ মহররম ১৪৩৩, মঙ্গলবার

কর্মশালায় ইউনিসেফের তথ্য

\section{মাতৃস্বাস্থ্যসেবায় বৈষম্য দেখা \\ দিয়েছে}

বিশেষ প্রতিনিধि •

হাসপাতালে ও সেবাকেন্দ্রে মাত্স্বাস্থ্যসেবায় বৈষম্য তৈরি হয়़ছছ।। গর্ভধারণ, প্রসবকাল ও প্রসব-পরবর্তী সেবা গ্রহণের হার দরিদ্রদের চেয়ে ধনীদের মট্যে বেশি। গতকাল সোমবার রাজধানীর একটি হোটেলে মাত ও নবজাতকের স্বাস্থ্য খাতে অর্থায়ন ও প্রনোদনা বিষয়ে আয়োজিত এক কর্মশালায় এ কথা বলা হয়। ইউনিলেফ, ইউরোপীয় ইউনিয়ন, ব্র্যাক বিশ্ববিদ্যালয়, ইউকে এইড $ও$ পপুলেশন কাউনিল যৌথভাবে দুদিটের এই কর্মশালার আয়োজন কটে।

প্রথম অধিটেশরের মৃল বক্তব্যে ইউনিসেফের কর্মকর্তা রিয়ৗদ মাহমুদ বরেন, সমাজের সবচ্য়য় দরিদ্র শ্রেণীর মায়েদর আট শতাংশ লেবাকেন্দ্রে সন্তান প্রসবের সুযোগ পান। সবচেয়ে ধনী শ্রেণীর মধ্যে এই হার ৫৩ শতাংশ। দরিদ্র শ্রেণীর নয় শতাংশ মা সন্তান জন্মদানের সময় দক্ষ স্বাস্থ্যকমীর সহায়তা পান। প্রসবপূর্ব (দরিদ্র ৩১ ও ধনী b-2 শতাংশ) ও প্রসব-পরবর্তী (দরিদ্র সাত ও ধনী ৫১ শতাংশ) লেবার ক্ষেত্রেও বৈষম্য রয়েছে। দরিদ্দদের ক্ষেত্রে অক্ততা, সামাজিক বিধিনিযেধ, ওষুধের দাম ও যাতায়াত খরচ সেবা পাওয়ার ক্ষেত্রে বাধা হয়ে দাঁড়ায়।

মা, নবজাতক \& শিফ স্বাস্থ্য সেবার উন্নয়নে স্বাস্থ্য মন্ত্রণালয় একাধিক জেলায় কর্মসম্পাদনাভিত্তিক প্রণোদনা (পে-ফর-পারফরম্যাস্স) ৪ চাহিদাভিতিক অর্থায়ন কর সৃচি (ডিম্যান্ড সাইড ফাইনাপ্গিং) চালু করেছে। ফলে সংশ্লিষ্ট জেলাগুনোতে মাত স্বাস্থ্যের উন্নতি হচ্ছে এবং মাতৃ ৫ শিশ মৃত্যুহার কমছে।

পপুলেশন কাউন্সিলের এ দেশের পরিচালক ওবায়দুর রব বলেন, ২০১৫ माলে মা ও শিশ স্বাস্থ্য সংশ্লিষ্ট সহয্রাব উন্নয়ন লক্ষ অর্জন নিয়ে সংশয় দেখ্খা দিলে পথিবীর বিভিন্ন দেণ কর্মসম্পাদনাভিত্তিক প্রটোদনা ও চাহিদাভিতিক অর্থায়ন কর্মসূচি চালু করে সুফল পাওয়া গেটছ। 


\section{श्ताक्णलाa}

\section{পারফরমেন্স ভিত্তিক প্রণোদনা স্বাস্থ্য সেবায় নতুন অধ্যায় সৃষ্টি করবে}

\section{বিলেশষজ্ঞদের অভিমত}

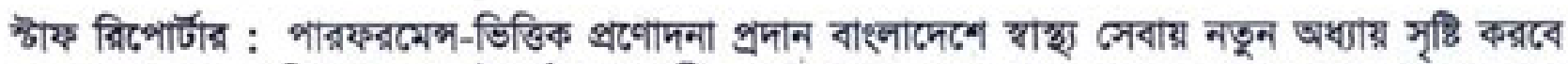
বলে মলে করেন বিশেষজ্ঞরা। টার্গেট অনুযায়ী সেবা প্রদান করলে হাসপাতাল থেকে সেবা গ্রহণের হার

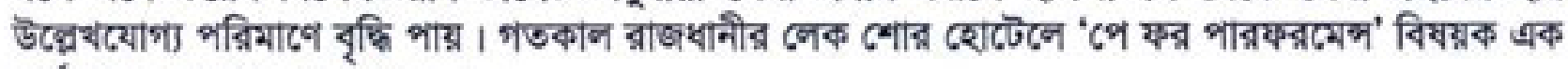
কর্মশালায় বক্তার্না এ কধা বলেन।

"পে ফ্র পার্নফ্রমেঙ্গ" হল সেবা প্রদানকার্রী প্রতিষ্ঠান এবং সেবা প্রদানকার্রীকে আর্থিক সুবিধা প্রদানের্ন মাধ্যমে কর্মদকতত এবং কর্মসমপাদনের হার বৃদ্ধিতে উৎসাহ দেয়া। পে ফ্র পাব্রফ্রমেন্স মডেলে প্রতিষ্ঠানকে প্রণোদনা প্রদানের মাধ্যমে তাদের পারফ্রমেন্স বৃদ্ছির প্রয়াস থাকে ফলে সেবাগ্বহীতাও মানসম্পন্ন সেবা পেয়ে থাকেন। বিশেষ করে দুর্গম ও পশাৎপদ এলাকা, লো-পারফর্মিং এলাকা, চর কিংবা হাওড় এলাকার্র জন্য এ মডেল খুবই ซর্রगত্তৃরূর।

স্বাস্য্য অধিদপ্তর এবং পপুলেশন কাউন্সিলের যৌধ উদ্যোগে আয়োজিত এ কর্মশালায় সভাপত্তিত্র করেনপ্রাইমারি হেলথ কেয়ারের্ন পর্নিচালক ডা. মোঃ শামসুল হক। কর্মশালায় পে ফ্র পারফ্ন্রমেন্স বিষয়ক

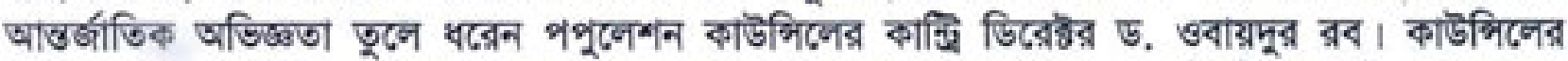

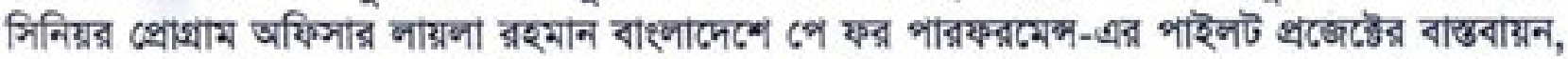
ফ্লাফল এবং অর্জিত অভিজ্ঞতার উপর্ন একটি তথ্যপূর্ণ প্রতিবেদন উপস্থাপন কর্রেন।

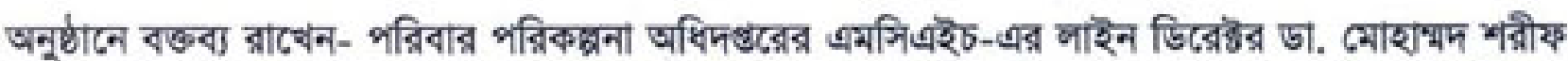

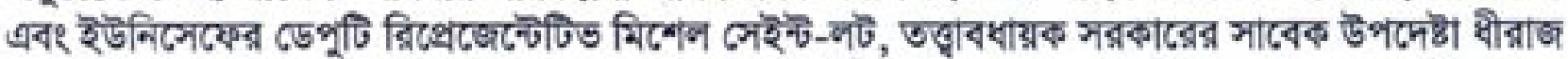
কুমাব্র নাধ, স্থাস্থ মস্ত্রণালয়ের হেলধ ইকোনোমিক্স ইউনিটের যুগ্মা প্রধান প্রশাত্ত ভূষণ বড়য়া, স্বাস্থ্য অধিদপ্তর্রের ডা. অািজ্রুল আলীম, ইউনিসেফের ড. রিয়াদ মাইমুদ এবং পপুলেশন কাউসিলের অ্যাডভাইজার একেএম खाएব্ন উল্পাহ थान।

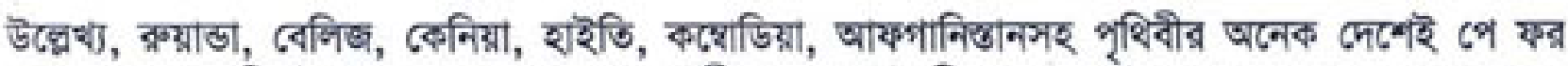
পারফন্নমেন্স প্রচলিত আছে। এর মাধ্যমে সেবাগ্রহীতার্র হার্র যেমনি বাড়বে, সাথে সাথে সেবার্র ওুণগত মানও বাড়বে। এত্ত সেবা প্রদানকারী এবং সেবা গ্রণকারী উভয়েই উপকৃত হবে। বাইলাদেশে এই পে ফর্র

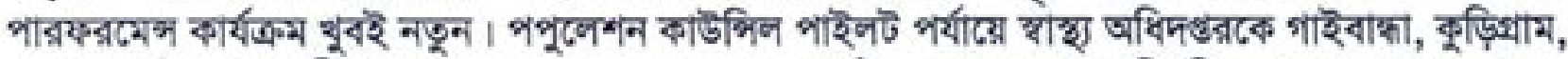

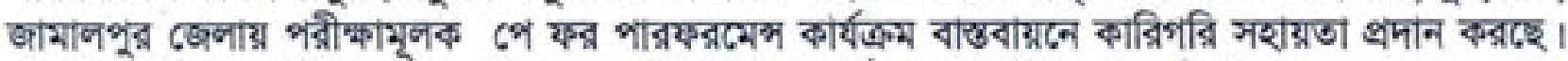
দুই দিনব্যাপী এ কর্মশালায় সরকারি-বেসরকারি বিভিন্ন সংগঠনের শতাধিক স্বাস্থ্য বিশেষজ্ঞ অংশগ্রহণ করেন। 


\section{(1) Population Council}

Population Council

Bangladesh Country Office

House CES (B) 21, Road 118, Gulshan

Dhaka, Bangladesh

www.popcouncil.org 